\title{
WEAK LIMITS OF PROJECTIONS AND COMPACTNESS OF SUBSPACE LATTICES
}

\author{
BRUCE H. WAGNER
}

\begin{abstract}
A strongly closed lattice of projections on a Hilbert space is compact if the associated algebra of operators has a weakly dense subset of compact operators. If the lattice is commutative, there are necessary and sufficient conditions for compactness, one in terms of the structure of the lattice, and the other in terms of a measure on the lattice. There are many examples of compact lattices, and two main types of examples of noncompact lattices. Compactness is also related to the study of weak limits of certain projections.
\end{abstract}

In [A3], Arveson generalized N. Andersen's important theorem on continuous nests [An, Theorem 3.5.5] to certain commutative subspace lattices associated with " $\Sigma$-continuous" measures. An important property of these lattices is that they are compact in the strong operator topology. Since compactness is also a property of nests, and plays an important role in the similarity theory for nests [Da2, La, An], we investigate in this paper the problem of determining which subspace lattices are compact. A greater understanding of this problem will hopefully help in the development of similarity theory for other lattices. It should be noted that it is well-known that there are also noncompact lattices, namely orthocomplemented commutative subspace lattices.

In §2, we give a main result (Theorem 2.2) which provides a connection between compactness of a lattice $\mathscr{L}$ and the presence of compact operators in the associated operator algebra alg $\mathscr{L}$. This result provides many examples of compact lattices. It also shows that there is a relationship between complete distributivity, compactness, and other nice properties, especially if the lattice is commutative.

$\$ 3$ deals with properties of noncompact lattices. Included are two conditions equivalent to noncompactness for commutative lattices: one is essentially measuretheoretic, and the other is a condition on the structure of the lattice. We also give two quite different classes of examples of noncompact lattices. In the process, we obtain a representation theorem for certain infinite tensor products of commutative subspace lattices, which extends a result in [GHL]. We then show that "most" infinite tensor products are noncompact.

One simple condition determining compactness is whether or not the lattice has any nonprojection weak limits (Proposition 2.1). This property is related to a paper

Received by the editors June 27, 1986. Some of the results in this paper were presented at the NSF-CBMS Conference on Quasitriangularity and Analyticity in Operator Algebras, Texas Tech University, August 1, 1983.

1980 Mathematics Subject Classification (1985 Revision). Primary 47D25. 
by Foias, Pasnicu, and Voiculescu [FPV] on weak limits of certain projections. We study this relationship in $\S 4$, and also derive other consequences from the existence of such weak limits. $\$ 4$ mostly deals with weak limits starting from the point of view of an operator algebra instead of a lattice, and is thus essentially self-contained.

The author would especially like to thank Cecelia Laurie, Robert L. Moore, John Froelich, William Arveson, Justin Peters, and Alan Hopenwasser for several valuable discussions and insights.

1. Preliminaries. Every Hilbert space in this paper will be complex, infinite-dimensional, and separable. The set of bounded operators on a Hilbert space $\mathscr{H}$ will be denoted by $\mathscr{B}(\mathscr{H})$ and the set of compact operators by $\mathscr{K}$. All operators discussed will be bounded, and all projections will be self-adjoint. If $P$ is a projection, then $P^{\perp}=I-P$.

If $\mathscr{L}$ is a collection of projections acting on $\mathscr{H}$, then we denote by alg $\mathscr{L}$ the set of operators which leave each projection in $\mathscr{L}$ invariant, i.e., alg $\mathscr{L}=\{T \in \mathscr{B}(\mathscr{H})$ : $P^{\perp} T P=0$ for all $P$ in $\left.\mathscr{L}\right\}$. alg $\mathscr{L}$ is an algebra which is closed in the weak operator topology. Similarly, if $\mathscr{A}$ is an algebra in $\mathscr{L}(\mathscr{H})$, then lat $\mathscr{A}=$ projections $P$ in $\mathscr{B}(\mathscr{H}): P^{\perp} T P=0$ for all $T$ in $\left.\mathscr{A}\right\}$. lat $\mathscr{A}$ is a lattice which is closed in the strong operator topology. In general, a subspace lattice is a lattice of projections which is strongly closed and which contains 0 and $I$. Every subspace lattice is complete (closed under arbitrary intersections and closed linear spans). A subspace lattice is commutative if all the projections mutually commute. In this case, $\mathscr{L}=$ lat(alg $\mathscr{L})[\mathbf{A 2}$, Theorem 1.6.3].

A projection $R$ is semi-invariant for a collection of operators $\mathscr{A}$ if $R=P^{\prime}-P$ for some $P, P^{\prime} \in$ lat $\mathscr{A}$ with $P^{\prime}>P$. If $\mathscr{A}$ is an algebra, then so is $R \mathscr{A} R[\mathbf{S}$, Lemma 0]. $R \mathscr{A} R$ is called a reduced algebra of $\mathscr{A}$. If $\mathscr{L}$ is a commutative subspace lattice and $\mathscr{A}=$ alg $\mathscr{L}$, then $R \mathscr{A} R$ is a subalgebra of $\mathscr{A}$ since $\mathscr{L} \subset$ alg $\mathscr{L}$.

Finally, if $\mathscr{L}$ is a commutative subspace lattice, then a semi-invariant projection $R$ for alg $\mathscr{L}$ is an atom of $\mathscr{L}$ if $R P=0$ or $R P=R$ for every $P \in \mathscr{L} . \mathscr{L}$ is nonatomic if it has no atoms, and is purely atomic if there are atoms $E_{1}, E_{2}, \ldots$ such that $\sum_{i=1}^{\infty} E_{i}=I$. Note that an atom is just a minimal projection in the abelian von Neumann algebra $\mathscr{L}^{\prime \prime}, \mathscr{L}$ is nonatomic iff $\mathscr{L}^{\prime \prime}$ is nonatomic, and $\mathscr{L}$ is purely atomic iff $\mathscr{L}^{\prime \prime}$ is generated by its minimal projections.

2. Compact lattices. In this section, $\mathscr{L}$ denotes a subspace lattice (i.e., always strongly closed). We will investigate the question of whether $\mathscr{L}$ is compact in the strong operator topology. Thus, " $\mathscr{L}$ is compact" will always mean $\mathscr{L}$ is strongly compact. (In fact, there is no ambiguity anyway, since the following proposition implies that strong compactness of $\mathscr{L}$ is equivalent to compactness of $\mathscr{L}$ in any of the weak, ultraweak, or ultrastrong topologies.) Proposition 2.1 was shown to the author by William Arveson.

Proposition 2.1. $\mathscr{L}$ is compact $\Leftrightarrow \mathscr{L}$ is weakly closed

Proof. $\Rightarrow$ Suppose $\mathscr{L}$ is strongly compact. Let $\alpha$ : ( $\mathscr{L}$, strong) $\rightarrow(\mathscr{L}$, weak) be the identity map. Since $\alpha$ is continuous, $\mathscr{L}$ is weakly compact and therefore weakly closed. 
$\Leftarrow$ If $\mathscr{L}$ is weakly closed, then $\mathscr{L}$ is weakly compact since it is contained in the unit ball of $\mathscr{B}(\mathscr{H})$. Now let $\left\{P_{n}: 1 \leqslant n<\infty\right\} \subseteq \mathscr{L}$. Then there is some subsequence $\left\{P_{n_{k}}\right\}$ such that $P_{n_{k}} \rightarrow$ some $P \in \mathscr{L}$ weakly. But since $P$ is a projection, and the strong and weak topologies agree on the set of projections, it follows that $P_{n_{k}} \rightarrow P$ strongly. Therefore, $\mathscr{L}$ is strongly compact (sequential compactness is sufficient since the unit ball of $\mathscr{B}(\mathscr{H})$ is metrizable in the strong operator topology).

We can now prove the main result of this section, which gives a connection between the compactness of $\mathscr{L}$ and the presence of compact operators in alg $\mathscr{L}$.

THEOREM 2.2. Let $\mathscr{A}=$ alg $\mathscr{L}$. If $R(\mathscr{A} \cap \mathscr{K}) R \neq\{0\}$ for every nonzero semiinvariant projection $R$ for $\mathscr{A}$, then $\mathscr{L}$ is compact.

Proof. If $\mathscr{L}$ is not compact, then Proposition 2.1 and the weak compactness of the unit ball of $\mathscr{B}(\mathscr{H})$ imply that there is some sequence $\left\{P_{n}: 1 \leqslant n<\infty\right\} \subseteq \mathscr{L}$ and a nonprojection $Q$ such that $P_{n} \rightarrow Q$ weakly. Note that $Q$ is a positive contraction. We first claim that the subspace $\overline{Q \mathscr{H}}$ is invariant for $\mathscr{A}$. To see this, let $k \in \operatorname{ker} Q=\operatorname{ker} \sqrt{Q}$. Then for any $h \in \mathscr{H}$ and $T \in \mathscr{A}$,

$$
\begin{aligned}
|\langle T Q h, k\rangle| & =\lim \left|\left\langle T P_{n} h, k\right\rangle\right|=\lim \left|\left\langle P_{n} T P_{n} h, k\right\rangle\right| \\
& =\lim \left|\left\langle T P_{n} h, P_{n} k\right\rangle\right| \leqslant \lim \|T\|\left\|P_{n} h\right\|\left\|P_{n} k\right\| \\
& =\|T\|\|\sqrt{Q} h\|\|\sqrt{Q} k\|=0 \\
& \Rightarrow T Q h \in \overline{Q^{*} \mathscr{H}}=\overline{Q \mathscr{H}} .
\end{aligned}
$$

Now $P_{n}{ }^{\perp} \rightarrow I-Q$ weakly and $\mathscr{A}^{*}=\operatorname{alg} \mathscr{L}^{\perp}$, so the above argument applied to $I-Q$ and $\mathscr{A}^{*}$ implies that $(I-Q) \mathscr{H}$ is invariant for $\mathscr{A}^{*}$. It follows that

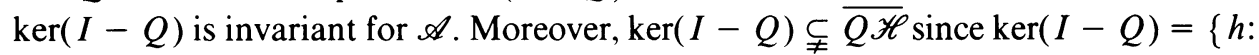
$Q h=h\}$ and $Q$ is not a projection.

Let $R$ be the projection onto $\overline{Q \mathscr{H}} \ominus \operatorname{ker}(I-Q)$. By the above, $R$ is a nonzero semi-invariant projection for $\mathscr{A}$. Let $K \in \mathscr{A} \cap \mathscr{K}$. Then $P_{n} K\left(P_{n}-Q\right) \rightarrow 0$ weakly since $K$ is compact. But

$$
\begin{aligned}
P_{n} K\left(P_{n}-Q\right) & =P_{n} K P_{n}-P_{n} K Q=K P_{n}-P_{n} K Q \\
& \rightarrow K Q-Q K Q=(I-Q) K Q \quad \text { weakly } \\
& \Rightarrow(I-Q) K Q=0 .
\end{aligned}
$$

Thus, $K(\overline{Q \mathscr{H}}) \subseteq \operatorname{ker}(I-Q)$, so $R(\mathscr{A} \cap \mathscr{K}) R=\{0\}$.

COROLlaRY 2.3. If $\mathscr{L}$ is commutative and every semi-invariant projection contains an atom, then $\mathscr{L}$ is compact.

Proof. If $E$ is an atom contained in a semi-invariant projection $R$ for $\mathscr{A}=\operatorname{alg} \mathscr{L}$, then $R(\mathscr{A} \cap \mathscr{K}) R \supseteq E(\mathscr{A} \cap \mathscr{K}) E \supseteq E\left(\mathscr{L}^{\prime} \cap \mathscr{K}\right) E=E \mathscr{K} E \neq\{0\}$.

COROllary 2.4. If $\mathscr{L}$ is commutative and purely atomic, then $\mathscr{L}$ is compact.

The previous two corollaries are special cases of a more general result (Corollary 2.6 below). A subspace lattice $\mathscr{L}$ (not necessarily commutative) is completely 
distributive if the identity

$$
\bigwedge_{i}\left(\bigvee_{j} P_{i j}\right)=\bigvee_{f \in J^{\prime}}\left(\bigwedge_{i} P_{i, f(i)}\right)
$$

and its dual hold for all choices of $P_{i j} \in \mathscr{L}(i \in I, j \in J)$, where $I$ and $J$ are any indexing sets and $J^{I}$ denotes the set of all functions $f: I \rightarrow J$. We need the following important result.

Theorem 2.5 (LAurie And LongstafF [LL, Lemma 2]). Let $\mathscr{L}$ be a subspace lattice and $\mathscr{A}=\operatorname{alg} \mathscr{L}$. Let $\mathscr{R}$ denote the rank-one subalgebra of $\mathscr{A}$ (the algebra generated by the rank-one operators in $\mathscr{A})$. Then $\mathscr{L}$ is completely distributive if and only if $R \mathscr{R} R \neq\{0\}$ for every nonzero semi-invariant projection $R$ for $\mathscr{A}$.

Since $R \mathscr{R} R \subseteq R(\mathscr{A} \cap \mathscr{K}) R$, we immediately obtain

Corollary 2.6. If $\mathscr{L}$ is completely distributive, then $\mathscr{L}$ is compact.

We note that nests (linearly ordered lattices) are completely distributive, as are the lattices which satisfy the hypotheses of Corollaries 2.3 and 2.4. Also, Laurie has shown [L2] that the $\sum$-continuous lattices introduced by Arveson [A3], which are compact, are also completely distributive. There is an example in [Lam, §3] of a noncommutative subspace lattice which is compact and not completely distributive, but for commutative subspace lattices the converse of Corollary 2.6 is unknown. It should be noted that in the commutative case there are other equivalent conditions for complete distributivity, due to Laurie and Longstaff [LL] (also, see [HLM]), and other conditions which are related to compactness. The situation is summarized by the following theorem. Here $\mathscr{C}_{p}$ denotes the Schatten $p$-class of compact operators.

TheOrem 2.7. Suppose $\mathscr{L}$ is a commutative subspace lattice and $\mathscr{A}=$ alg $\mathscr{L}$. Consider the following properties:

(a) $\mathscr{L}$ is completely distributive.

(b) For some $p \leqslant 2, R\left(\mathscr{C}_{p} \cap \mathscr{A}\right) R \neq\{0\}$ for every nonzero semi-invariant projection $R$ for $\mathscr{A}$.

$\left(b^{\prime}\right)\left(\mathscr{C}_{p} \cap \mathscr{A}\right)$ is dense in $\mathscr{A}$ for some $p \leqslant 2$ in any of the strong, ultrastrong, weak, or ultraweak topologies.

(c) $R(\mathscr{A} \cap \mathscr{K}) R \neq\{0\}$ for every nonzero semi-invariant projection $R$ for $\mathscr{A}$.

(c') $\mathscr{A} \cap \mathscr{K}$ is dense in $\mathscr{A}$ in any of the strong, ultrastrong, weak, or ultraweak topologies.

(d) $\mathscr{L}$ is compact.

(e) $\mathscr{A}+\mathscr{K}$ is norm-closed.

Then (a) $\Leftrightarrow(\mathrm{b}) \Leftrightarrow\left(\mathrm{b}^{\prime}\right) \Rightarrow(\mathrm{c}) \Leftrightarrow\left(\mathrm{c}^{\prime}\right) \Rightarrow(\mathrm{d})$ and $(\mathrm{e})$.

Proof. The equivalence of $(a),(b)$, and $\left(b^{\prime}\right)$ was proved in $[\mathbf{L L}]$.

$(c) \Leftrightarrow\left(c^{\prime}\right)$ : This follows the argument given for $(b) \Leftrightarrow\left(b^{\prime}\right)$. For $(c) \Rightarrow\left(c^{\prime}\right)$, Theorem 4.1 of [L1] implies ultraweak density. Density in the other topologies follows since $\mathscr{A} \cap \mathscr{K}$ is convex. For $\left(\mathrm{c}^{\prime}\right) \Rightarrow(\mathrm{c})$, density implies that there is a net $\left\{T_{\alpha}\right\} \subseteq$ $\mathscr{A} \cap \mathscr{K}$ such that $T_{\alpha} \rightarrow I$ in the appropriate topology. Hence, for any nonzero projection $P, P T_{\alpha} P \rightarrow P \neq 0$, so there is some $T \in \mathscr{A} \cap \mathscr{K}$ with $P T P \neq 0$. 
(b) $\Rightarrow$ (c): Clear.

(c) $\Rightarrow(\mathrm{d})$ : Theorem 2.2 .

$\left(c^{\prime}\right) \Rightarrow(e)$ : Theorem 1.1 of [FAM].

Note. The implication (a) $\Rightarrow$ (e) was proved by Laurie in [L1, Corollary 4.3]. Also, observe that if $(b)$ or $\left(b^{\prime}\right)$ holds, then Theorem 2.5 implies that the same condition holds for all $p$.

REMARKS. (e) $\nRightarrow$ (c) since if $\mathscr{L}$ is nonatomic and orthocomplemented, then $\mathscr{A}=\mathscr{L}^{\prime}$, and it is well known that $\mathscr{L}^{\prime} \cap \mathscr{K}=\{0\}$. But $\mathscr{L}^{\prime}+\mathscr{K}$ is norm-closed since $\mathscr{L}^{\prime}$ is a $C^{*}$-algebra. Since such a lattice $\mathscr{L}$ is not compact (see Theorem 3.2), it also follows that $(e) \nRightarrow(d)$.

It is unknown whether or not $(d) \Rightarrow(e),(d) \Rightarrow(c)$, or $(c) \Rightarrow(b)$. The material in $\$ 4$ shows some of the problems involved in proving or disproving $(d) \Rightarrow(c)$. In regard to the implication (c) $\Rightarrow(b)$, Froelich has given a number of examples in [F2] of commutative subspace lattrices $\mathscr{L}$ with $\mathscr{A}=$ alg $\mathscr{L}$ containing certain types of compact operators, but not others. For instance, one such example has $\mathscr{A} \cap \mathscr{K} \neq\{0\}$ but $\mathscr{A} \cap \mathscr{C}_{p}=\{0\}$ for all $p<\infty$. But none of these examples has the property (c') that $\mathscr{A} \cap \mathscr{K}$ is ultraweakly dense in $\mathscr{A}$. There is also an example in [F2] of a commutative subspace lattice $\mathscr{L}$ with alg $\mathscr{L}+\mathscr{K}$ not norm-closed. But in this example $\mathscr{L}$ is also not compact.

3. Noncompact lattices. In this section, we give some examples of noncompact subspace lattices. These fall into two basic types: some "piece" of the lattice is either a nonatomic Boolean algebra or a certain infinite tensor product of commutative subspace lattices. This will be made precise below. In addition, after obtaining a partial representation theorem for infinite tensor products of commutative subspace lattices, we show that "most" of them are noncompact. We will also give two conditions which are equivalent to noncompactness for commutative subspace lattices. One is essentially measure-theoretic, and the other is lattice-theoretic. Theorem 2.2 gives only a necessary condition for noncompactness, and this is an algebraic condition. Finally, we also prove that locally compact commutative lattices must actually be compact. Every lattice in this section will be a commutative subspace lattice, often abbrieviated CSL.

Lemma 3.1. Suppose that $\mathscr{L}$ is commutative, and suppose that $\rho$ is a faithful normal state on $\mathscr{L}^{\prime \prime}$. Then $\mathscr{L}$ is not compact if and only if there is an $\varepsilon>0$ and a sequence $\left\{P_{n}: 1 \leqslant n<\infty\right\} \subseteq \mathscr{L}$ such that $\rho\left(P_{n} \Delta P_{m}\right) \geqslant \varepsilon$ for all $n \neq m$, where $P_{n} \Delta P_{m}=$ $P_{n} \vee P_{m}-P_{n} \wedge P_{m}=P_{n}+P_{m}-2 P_{n} P_{m}$.

Proof. $\Leftarrow$ If $\left\{P_{n}\right\}$ has a strongly convergent subsequence $\left\{P_{n_{k}}\right\}$, then $P_{n_{k}}-P_{n_{j}}$ $\rightarrow 0$ strongly as $k, j \rightarrow \infty$, which is equivalent to $\rho\left(\left(P_{n_{k}}-P_{n_{j}}\right)\left(P_{n_{k}}-P_{n_{j}}\right)\right) \rightarrow 0$ [Di, I.4.6. Proposition 4]. But $\left(P_{n_{k}}-P_{n_{j}}\right)\left(P_{n_{k}}-P_{n_{j}}\right)=P_{n_{k}}+P_{n_{j}}-2 P_{n_{k}} P_{n_{j}}=$ $P_{n_{k}} \Delta P_{n_{1}}$ and $\rho\left(P_{n_{k}} \Delta P_{n_{j}}\right) \geqslant \varepsilon$ for all $k \neq j$.

$\Rightarrow$ If $\mathscr{L}$ is not compact, then there is a sequence $\left\{P_{n}: 1 \leqslant n<\infty\right\}$ of distinct projections in $\mathscr{L}$ with no strongly convergent subsequence. It follows that there is an $\varepsilon>0$ such that $\rho\left(\left(P_{n}-P_{m}\right)\left(P_{n}-P_{m}\right)\right) \geqslant \varepsilon$ for all $n \neq m$, i.e., $\rho\left(P_{n} \Delta P_{m}\right) \geqslant \varepsilon$. 
The following theorem is well known. The standard proof uses Liapounoff's theorem [R, Theorem 5.5] to give the stronger result that the weak closure of $\mathscr{L}$ is $\left\{\mathscr{A} \in \mathscr{L}^{\prime \prime}: 0 \leqslant A \leqslant 1\right\}$. We give an elementary proof using Lemma 3.1.

THEOREM 3.2. Suppose $\mathscr{L}$ is a nonatomic Boolean algebra (i.e., $\mathscr{L}$ is orthocomplemented and commutative). Then $\mathscr{L}$ is not compact.

Proof. Since $\mathscr{L}$ is nonatomic, it must contain a nonatomic nest $\mathscr{P}$. If $\rho$ is any faithful normal state on $\mathscr{L}^{\prime \prime}$, then $\rho: \mathscr{P} \rightarrow[0,1]$ is bijective. Let $P_{t}=\rho^{-1}(t)$. Since $\mathscr{P}{ }^{\perp} \subset \mathscr{L}, P_{t}-P_{s} \in \mathscr{L}$ for all $t>s$. Define $P[s, t]=P_{t}-P_{s}$ for $s<t$. Now let $Q_{1}=P[0,1 / 2], \quad Q_{2}=P[0,1 / 4]+P[1 / 2,3 / 4], \quad Q_{3}=P[0,1 / 8]+P[1 / 4,3 / 8]+$ $P[1 / 2,5 / 8]+P[3 / 4,7 / 8]$, etc. In general,

$$
Q_{n}=\sum_{k=1}^{2^{n-1}} P\left[\frac{2 k-2}{2^{n}}, \frac{2 k-1}{2^{n}}\right] .
$$

Then each $Q_{n} \in \mathscr{L}$ and $\rho\left(Q_{n} \Delta Q_{m}\right)=\frac{1}{2}$ for all $n \neq m$, so Lemma 3.1 implies that $\mathscr{L}$ is not compact.

Definition. Suppose that $\mathscr{L}$ is commutative, and let $S$ be a projection in $\mathscr{L}^{\prime}$. Then $\mathscr{L}^{S}=S \mathscr{L}$, acting on $S \mathscr{H}$, is a subspace lattice, called an induced lattice of $\mathscr{L}$. Note that $\operatorname{alg} \mathscr{L}^{S}=\left.S(\operatorname{alg} \mathscr{L})\right|_{S \mathscr{H}}$. As a special case, if $R$ is a semi-invariant projection for alg $\mathscr{L}$ (so $R \in \mathscr{L}^{\prime \prime} \subseteq \mathscr{L}^{\prime}$ ). then $\mathscr{L}^{R}$ is called a reduced lattice of $\mathscr{L}$, and will instead be denoted by $\mathscr{L}_{R}$.

Proposition 3.3. (a) If some induced lattice $\mathscr{L}^{S}$ is noncompact, then $\mathscr{L}$ is noncompact.

(b) If some strongly closed sublattice of $\mathscr{L}$ is noncompact, then $\mathscr{L}$ is noncompact.

(c) If some reduced lattice $\mathscr{L}_{R}$ is noncompact, then $\mathscr{L}$ is noncompact.

Proof. (b) is clear. For (a) and (c), if $\mathscr{L}$ is compact then so is $\mathscr{L}^{S}$ since the map $P \rightarrow S P S$ is strongly continuous.

Thus, if some "piece" of $\mathscr{L}$ is noncompact, so is $\mathscr{L}$.

COROLLARY 3.4. If some reduced lattice $\mathscr{L}_{R}$ or induced lattice $\mathscr{L}^{S}$ has a strongly closed sublattice $\tilde{\mathscr{L}}$ which is nonatomic and orthocomplemented, then $\mathscr{L}$ is noncompact.

We can now completely determine the compactness of orthocomplemented CSLs.

COROLlaRY 3.5. If $\mathscr{L}$ is commutative and orthocomplemented, then $\mathscr{L}$ is compact if and only if $\mathscr{L}$ is purely atomic.

Proof. $\Rightarrow$ Let $P$ be the sum of the atoms of $\mathscr{L}$. Note that $P \in \mathscr{L}$. If $\mathscr{L}$ is not purely atomic, then $P \neq I$. In this case, let $R=I-P$. Then $\mathscr{L}_{R}$ is nonatomic and orthocomplemented, and thus is noncompact by Theorem 3.2. Proposition 3.3 then implies that $\mathscr{L}$ is noncompact.

$\Leftarrow$ Follows from Corollary 2.4 .

Although it is not necessary for the next result, it will be convenient to use the Arveson representation for CSLs [A2, Theorem 1.3.1]. This representation is also often useful for finding examples of CSLs which have certain properties, and will 
prove invaluable in our study of infinite tensor products. Given any commutative subspace lattice $\mathscr{L}$, the Arveson representation states that there is a partially ordered measure space $(X, \leqslant, \mu)$ such that $\mathscr{L}$ is unitarily equivalent to a certain lattice denoted by $\mathscr{L}(X, \leqslant, \mu)$. Here $X$ is a compact metric space, $\leqslant$ is a reflexive and transitive relation on $X$ whose graph $G$ is closed in $X \times X$, and $\mu$ is a Borel probability measure on $X$. A Borel set $E \subseteq X$ is increasing if $x \in E$ and $x \leqslant y \Rightarrow y$ $\in E$. Finally, $\mathscr{L}(X, \leqslant, \mu)=\left\{P_{E}: E \subseteq X\right.$ is an increasing Borel set $\}$, where $P_{E}$ is the multiplication operator acting on $L^{2}(X, \mu)$ by $\left(P_{E} f\right)(x)=\chi_{E}(x) f(x)$.

Now if $\rho$ is a faithful normal state on $\mathscr{B}\left(L^{2}(X, \mu)\right)$, we can write $\rho(T)=$ $\sum_{i=1}^{\infty}\left\langle T f_{i}, f_{i}\right\rangle$, where $\sum_{i=1}^{\infty}\left\|f_{i}\right\|^{2}=1$, the $f_{i}$ 's are mutually orthogonal, and $\operatorname{span}\left\{f_{i}: 1 \leqslant i<\infty\right\}$ is dense in $L^{2}(X, \mu)$. It follows that if $\left\{P_{E_{i}}: 0 \leqslant i<\infty\right\} \subseteq$ $\mathscr{L}(X, \leqslant, \mu)$, then $P_{E_{n}} \rightarrow P_{E_{0}}$ strongly if and only if $\rho\left(P_{E_{n}} \Delta P_{E_{0}}\right) \rightarrow 0$ if and only if $\mu\left(E_{n} \Delta E_{0}\right) \rightarrow 0$. This shows that Lemma 3.1 is really a measure-theoretic condition for noncompactness. The author would like to thank Cecelia Laurie for pointing out the above measure condition.

We will now give a purely lattice-theoretic condition. This condition was used by Froelich in [F1] to show that alg $\mathscr{L}$ for a certain commutative lattice $\mathscr{L}$ has no compact operators. Note the connection with Theorem 2.2 (but notice also that Theorem 2.2 applies in the noncommutative case as well).

Theorem 3.6 (Froelich, R. L. Moore, And Wagner). A CSL $\mathscr{L}$ is noncompact $\Leftrightarrow$ there is a reduced lattice $\mathscr{L}_{R}$ and a sequence $\left\{P_{n}: 1 \leqslant n<\infty\right\} \subseteq \mathscr{L}_{R}$ such that

$$
\bigvee_{k} P_{n_{k}}=\left.I\right|_{R \mathscr{H}} \quad \text { and } \bigwedge_{k} P_{n_{k}}=\left.0\right|_{R \mathscr{H}}
$$

for every subsequence $\left\{P_{n_{k}}\right\}$.

Proof. $\Rightarrow$ If $\mathscr{L}$ is noncompact, then there is a sequence $\left\{P_{n}^{\prime}: 1 \leqslant n<\infty\right\} \subseteq \mathscr{L}$ and a nonprojection $Q$ such that $P_{n}^{\prime} \rightarrow Q$ weakly. As shown in the proof of Theorem 2.2, $\operatorname{ker}(I-Q)$ and $\overline{Q \mathscr{H}}$ are invariant subspaces for alg $\mathscr{L}$, and $\operatorname{ker}(I-Q) \subsetneq \overline{Q \mathscr{H}}$. Thus, if $N$ and $M$ are the projections onto $\operatorname{ker}(I-Q)$ and $\overline{Q \mathscr{H}}$, respectively, then $R=M-N$ is semi-invariant for $\operatorname{alg} \mathscr{L} . N, M \in \mathscr{L}$ since $\mathscr{L}$ is commutative, and therefore $R P_{n}^{\prime}=R P_{n}^{\prime} R=P_{n}^{\prime} R$ is a projection. It follows that $R P_{n}^{\prime} \rightarrow R Q$ weakly and $R Q=R Q R=Q R$.

Now restrict attention to $R \mathscr{H}$. Let $Q_{R}=\left.Q\right|_{R \mathscr{H}}$ and $P_{n}=\left.P_{n}^{\prime}\right|_{R \mathscr{H}}$. By the above, $P_{n} \rightarrow Q_{R}$ weakly, $\operatorname{ker}\left(I-Q_{R}\right)=\{0\}$, and $\operatorname{ker}\left(Q_{R}\right)=\overline{Q_{R}(R \mathscr{H})}{ }^{\perp}=\{0\}$. Now suppose $E=\wedge_{k} P_{n_{k}} \neq 0$ for some subsequence $\left\{P_{n_{k}}: 1 \leqslant k<\infty\right\}$. Then $0=$ $\left\langle P_{n_{k}}{ }^{1} E x, y\right\rangle \rightarrow\left\langle\left(I-Q_{R}\right) E x, y\right\rangle$ for every $x, y \in R \mathscr{H}$, so $\left(I-Q_{R}\right) E=0$ and thus $E=0$, a contradiction. Similarly, if $F=\mathrm{V}_{k} P_{n_{k}} \neq I$ for some subsequence, then $0=\left\langle P_{n_{k}} F^{\perp} x, y\right\rangle \rightarrow\left\langle Q_{R} F^{\perp} x, y\right\rangle$ for every $x, y \in R \mathscr{H}$, so $Q_{R} F^{\perp}=0$ and thus $F^{\perp}=0$, a contradiction.

$\Leftarrow$ By Proposition 3.3, if $\mathscr{L}_{R}$ is noncompact, then so is $\mathscr{L}$. Thus, we may assume without loss of generality that $R=I$. Using the Arveson representation, we have $\mathscr{L} \cong \mathscr{L}(X, \leqslant, \mu)$, and the hypothesis is equivalent to the existence of a sequence of increasing sets $\left\{E_{n}: 1 \leqslant n<\infty\right\}$ such that $\cup_{k} E_{n_{k}}=X$ a.e. $[\mu]$ and $\cap_{k} E_{n_{k}}=\varnothing$ a.e. $[\mu]$ for every subsequence $\left\{E_{n_{k}}\right\}$. It is then sufficient to show that there is no 
subsequence $\left\{E_{n_{\star}}\right\}$ which converges in measure, i.e., there is no increasing set $E$ such that $\mu\left(E_{n_{k}} \Delta E\right) \rightarrow 0$.

Suppose $\mu\left(E_{n_{k}} \Delta E\right) \rightarrow 0$, and assume first that $\mu(E) \neq 0$. Let $\varepsilon=\mu(E) / 2$, and let $F_{k}=E_{n_{k}}$ for convenience. Also, if $S$ is a subset of $X$, let $S^{c}$ denote the complement of $S$ in $X$. It follows that there is a subsequence $\left\{F_{k_{m}}: 1 \leqslant m<\infty\right\}$ such that $\mu\left(F_{k_{m}} \Delta E\right)<\varepsilon / 2^{m}$. Then $\bigcap_{m} F_{k_{m}}=\varnothing$ a.e., so

$$
2 \varepsilon=\mu\left(E \cap\left(\bigcap_{m} F_{k_{m}}\right)^{c}\right)=\mu\left(\bigcup_{m}\left(E \cap F_{k_{m}}^{c}\right)\right) \leqslant \sum_{m=1}^{\infty} \mu\left(F_{k_{m}} \Delta E\right)<\varepsilon,
$$

a contradiction. Now if instead $\mu(E)=0$, then $\mu\left(E_{n_{k}}^{c} \Delta E^{c}\right) \rightarrow 0$, and we can apply the above argument, replacing $F_{k_{m}}$ by $F_{k_{m}}^{c}$ and $E$ by $E^{c}$, to again get a contradiction since $\bigcap_{m} F_{k_{m}}^{c}=\left(\bigcup_{m} F_{k_{m}}\right)^{c}=\varnothing$ a.e.

REMARK. The preceding argument can be given without using the Arveson representation. Just replace increasing sets by projections in $\mathscr{L}, \mu$ by a faithful normal state $\rho, \cup$ by $\vee, \cap$ by $\wedge$, and set complement by orthogonal complement.

The theory developed in [A3], which motivated this study, is valid for representations of locally compact $*$-semigroups. A locally compact commutative subspace lattice is an example of such a $*$-semigroup, and one of the applications in [A3] dealt with the special case of certain compact CSLs. The next result shows that, if one wants to apply this theory to lattices, then there is no loss of generality in restricting to the compact case.

THEOREM 3.7. Every locally compact CSL is compact.

Proof. Suppose $\mathscr{L}$ is a locally compact CSL which is not compact. Then as in the proof of Theorem 3.6, there is a sequence $\left\{P_{n}: 1 \leqslant n<\infty\right\}$ in $\mathscr{L}$ and a nonprojection $Q$ such that $P_{n} \rightarrow Q$ weakly, and the projections $N$ and $M$ onto $\operatorname{ker}(I-Q)$ and $\overline{Q \mathscr{H}}$, respectively, are elements of $\mathscr{L}$ with $N<M$. It also follows from the proof of Theorem 2.2 that $(I-Q) K Q=0$ for every $K \in(\operatorname{alg} \mathscr{L}) \cap \mathscr{K}$, so $\mathscr{L}$ has no atoms $S$ such that $S \leqslant M-N$ (see the proof of Corollary 2.3). Therefore, there is a sequence $\left\{R_{j}: 1 \leqslant j<\infty\right\}$ in $\mathscr{L}$ such that $N<R_{j+1}<R_{j}<M$ for all $j$ and $R_{j} \rightarrow N$ strongly.

Since $\mathscr{L}$ is locally compact in the strong operator topology, $N$ has a neighborhood $\mathscr{N}$ with compact strong closure $\bar{N}$. The proof of Proposition 2.1 shows that $\overline{\mathcal{N}}$ is in fact weakly closed. Also, $\mathscr{N}$ must contain a set $\mathscr{U}=\left\{P \in \mathscr{L}:\left\|P h_{i}-N h_{i}\right\|\right.$ $<\varepsilon, 1 \leqslant i \leqslant m\}$ for some $\varepsilon>0$ and some $h_{1}, \ldots, h_{m}$ in $\mathscr{H}$, since the sets of this form are a base for the strong topology on $\mathscr{L}$. Now choose $J$ such that $R_{J} \in \mathscr{U}$, and let $R=R_{J}-N$. Then $N+P_{n} R \in \mathscr{L}$ and $N+P_{n} R \rightarrow N+Q R=Q R_{J}$ weakly. But $Q R_{J}$ is a nonprojection and $N+P_{n} R \in \mathscr{U}$ for all $n$ since

$$
\left\|\left(N+P_{n} R\right) h_{i}-N h_{i}\right\|=\left\|P_{n} R h_{i}\right\| \leqslant\left\|R h_{i}\right\|=\left\|R_{J} h_{i}-N h_{i}\right\|<\varepsilon
$$

for all $i$. This contradicts the fact that $\overline{\mathcal{N}}$ is weakly closed.

We conclude this section with a discussion of another type of noncompact lattice, quite different from that given by Theorem 3.2 and Corollary 3.4. This type consists of certain infinite tensor products of lattices. 
ExAmPLE. Let $X_{i}=\{0,1\}$ with the partial order $0 \leqslant 0 \leqslant 1 \leqslant 1$, and let $m_{i}$ be a probability measure on $X_{i}$ with $m_{i}(\{1\})=p_{i}, 0<p_{i}<1$. Then consider $\mathscr{L}=$ $\mathscr{L}(X, \leqslant, m)$, where $X=\prod_{i=1}^{\infty} X_{i}$, $\leqslant$ is the product ordering on $X\left(\left(x_{i}\right)_{i=1}^{\infty} \leqslant\right.$ $\left(y_{i}\right)_{i=1}^{\infty} \Leftrightarrow x_{i} \leqslant y_{i}$ for all $\left.i\right)$, and $m$ is the product measure $\prod_{i=1}^{\infty} m_{i}$. Finally, let $F_{k}=\left\{\left(x_{i}\right)_{i=1}^{\infty} ; x_{k}=1\right\} .\left\{F_{k}: 1 \leqslant k<\infty\right\}$ generates $\mathscr{L}$ as a subspace lattice by [A2, Theorem 1.2.2]. Here we have for convenience identified increasing sets with their corresponding projections, and we will continue to do so. Also, $\left(\omega_{i}\right)$ will always denote a sequence of 0 's and 1 's.

Case $1 . \prod_{i=1}^{\infty} P_{i}>0$ if and only if $\sum_{i=1}^{\infty}\left(1-p_{i}\right)$ converges. Therefore, if either $\sum p_{i}$ or $\sum\left(1-p_{i}\right)$ converges, then $\mathscr{L}$ has atoms. In fact, $\mathscr{L}$ is purely atomic and thus compact by Corollary 2.4. For example, if $\Pi p_{i}>0$, let $J$ denote any finite subset of $\mathbf{N}$ and define $A_{J}=\left\{\left(x_{k}\right) \in X: x_{k}=0\right.$ for all $k \in J, x_{k}=1$ for all $\left.k \notin J\right\}$, and $A_{0}=\left\{\left(x_{k}\right) \in X: x_{k}=1\right.$ for all $\left.k\right\}$. Then $A_{0}$ and each $A_{J}$ correspond to atoms of $\mathscr{L}$ and $I=A_{0}+\sum_{J} A_{J}$.

More generally, $\mathscr{L}$ has an atom if and only if there is a sequence $\left(y_{i}\right) \in X$ which has positive measure $\left(m\left(\left\{\left(x_{i}\right) \in X: x_{i}=y_{i}\right\}\right)>0\right)$. Thus, $\mathscr{L}$ has an atom if and only if $\sum\left|\omega_{i}-p_{i}\right|$ converges for some sequence $\left(\omega_{i}\right)$ of 0 's and 1's. Again, as above, it can be shown that in fact $\mathscr{L}$ is purely atomic in this case.

Case 2. If $\sum\left|\omega_{i}-p_{i}\right|$ does not converge for every $\left(\omega_{i}\right)$, then $\mathscr{L}$ is nonatomic. In particular, this will happen if there is some $\varepsilon>0$ such that $p_{i} \geqslant \varepsilon$ and $1-p_{i} \geqslant \varepsilon$ for all $i$. Then for $i \neq j$ we have

$$
\begin{aligned}
m\left(F_{i} \Delta F_{j}\right)= & m\left(\left\{\left(x_{k}\right) \in X: x_{i} \neq x_{j}\right\}\right) \\
= & m\left(\left\{\left(x_{k}\right) \in X: x_{i}=1 \text { and } x_{j}=0\right\}\right. \\
& \left.\quad \cup\left\{\left(x_{k}\right) \in X: x_{i}=0 \text { and } x_{j}=1\right\}\right) \\
= & p_{i}\left(1-p_{j}\right)+p_{j}\left(1-p_{i}\right)=p_{i}+p_{j}-2 p_{i} p_{j} \\
= & \left(\sqrt{p_{i}}-\sqrt{p_{j}}\right)^{2}+2 \sqrt{p_{i}} \sqrt{p_{j}}\left(1-\sqrt{p_{i}} \sqrt{p_{j}}\right) \\
\geqslant & 2 \sqrt{p_{i}} \sqrt{p_{j}}\left(1-\sqrt{p_{i}} \sqrt{p_{j}}\right) \\
\geqslant & 2 \varepsilon^{2} \quad \text { since } \sqrt{p_{i}} \sqrt{p_{j}} \leqslant 1-\varepsilon .
\end{aligned}
$$

It follows that $\mathscr{L}$ is not compact by Lemma 3.1 and the remarks preceding Theorem 3.6.

Case 3. Finally, suppose $\Sigma\left|\omega_{i}-p_{i}\right|$ does not converge for every $\left(\omega_{i}\right)$, but that $\left\{p_{i}\right\}$ is not bounded away from 0 and/or 1 . Partition $\mathbf{N}$ into an infinite number of infinite subsets $S_{1}, S_{2}, \ldots$ such that for each $j, \sum_{i \in S_{i}}\left|\omega_{i}-p_{i}\right|$ does not converge for every $\left(\omega_{i}\right)_{i \in s_{i}}$. This can be done as follows: successively find $N_{1}<N_{2}<N_{3}<\cdots$ such that for each $j, \sum_{i=N_{,-1}+1}^{N_{j}}\left|\omega_{i}-p_{i}\right| \geqslant 1$ for every $\left(\omega_{i}\right)_{i=N_{i-1}+1}^{N_{j}}$. Let $S_{1}=$ $\bigcup_{k=0}^{\infty}\left\{N_{2 k}+1, \ldots, N_{2 k+1}\right\}$ (where $N_{0}=0$ ) and $S_{2}^{\prime}=\bigcup_{k=0}^{\infty}\left\{N_{2 k+1}+1, \ldots, N_{2 k+2}\right\}$. Now similarly divide $S_{2}^{\prime}$ into two parts $S_{2}$ and $S_{3}^{\prime}$, divide $S_{3}^{\prime}$ into two parts $S_{3}$ and $S_{4}^{\prime}$, etc. If we let $\mathscr{L}_{i}$ be the sublattice of $\mathscr{L}$ generated by $\left\{F_{n}: n \in S_{i}\right\}$, then each $\mathscr{L}_{i}$ is nonatomic, and thus contains some increasing set $G_{i}$ with $m\left(G_{i}\right)=\frac{1}{2}$. The 
sequence $\left\{G_{i}: 1 \leqslant i<\infty\right\}$ has the property that $m\left(G_{i} \Delta G_{j}\right)=\frac{1}{2}$ for all $i \neq j$ (this is essentially the same calculation as in Case 2, with $p_{i}=p_{j}=\frac{1}{2}$ ), so again $\mathscr{L}$ is not compact.

Note that Case 2 is a special case of Case 3, but Case 2 with $p_{i}=p_{1}$ for all $i$ is really the basic example which allows one to analyze the more general situation. In fact, one can use this example to obtain the following:

Let $\mathscr{L}_{1}, \mathscr{L}_{2}, \ldots$ be nonatomic CSLs with Arveson representations $\mathscr{L}_{i} \cong$ $\mathscr{L}\left(X_{i}, \leqslant{ }_{i}, \mu_{i}\right)$ and $\mu_{i}\left(X_{i}\right)=1$ for all $i$. Now let $X=\prod_{i=1}^{\infty} X_{i}$, $\leqslant$ be the product ordering on $X, \mu=\prod_{i=1}^{\infty} \mu_{i}$, and let $\mathscr{L}=\mathscr{L}(X, \leqslant, \mu)$. In general (even if the $\mathscr{L}_{i}$ 's have atoms), we will call a lattice $\mathscr{L}$ formed in this way an infinite product lattice. Since each $\mathscr{L}_{i}$ is nonatomic, there are sets $E_{i} \in \mathscr{L}\left(X_{i}, \leqslant_{i}, \mu_{i}\right)$ with $\mu_{i}\left(E_{i}\right)=\frac{1}{2}$. Let $F_{i}=\left\{\left(x_{n}\right) \in X: x_{i} \in E_{i}\right\} \in \mathscr{L}$. Then $\mu\left(F_{i} \triangle F_{j}\right)=\frac{1}{2}$ for all $i \neq j$, so $\mathscr{L}$ is not compact. Note that if $\mathscr{L}$ were a finite product lattice $\left(X=\prod_{i=1}^{n} X_{i}\right.$, etc.), then we would have that $\mathscr{L} \cong \otimes_{i=1}^{n} \mathscr{L}_{i}$ [GHL]. In fact, this is true for infinite product lattices as well. We will prove this result and a partial converse below (Theorem 3.8), and then use it to obtain a general result on compactness of infinite tensor products of CSLs.

We first need some preliminaries on infinite tensor products. For each $i=1,2, \ldots$, let $a_{i}$ be a unit vector in a separable Hilbert space $\mathscr{H}_{i}$, and let $a=\left(a_{i}\right)_{i=1}^{\infty}$. $\mathscr{H}=\bigotimes_{i=1}^{x_{a}} \mathscr{H}_{i}$ is formed as in $[\mathbf{G}]$ or $[\mathbf{v N}]$ by completing the pre-Hilbert space consisting of finite linear combinations of elements of the form $\otimes_{i=1}^{\infty} h_{i}$ with $h_{i}=a_{i}$ for almost every $i$ (i.e., for all but a finite number), where the inner product on such elements is given by $\left\langle\otimes h_{i}, \otimes k_{i}\right\rangle=\prod_{i=1}^{\infty}\left\langle h_{i}, k_{i}\right\rangle$. If $\mathscr{R}_{i}$ is a von Neumann algebra acting on $\mathscr{H}_{i}$, then $\otimes_{i=1}^{\infty_{a}} \mathscr{R}_{i}$ is defined to be the von Neumann algebra on $\mathscr{H}$ generated by operators of the form $\otimes_{i=1}^{\infty} T_{i}$ with $T_{i} \in \mathscr{R}_{i}$ for all $i$ and $T_{i}=I$ for almost every $i$. In particular, $\mathscr{B}(\mathscr{H})=\bigotimes_{i=1}^{\infty_{a}} \mathscr{B}\left(\mathscr{H}_{i}\right)$ (see $[\mathbf{G}]$ for details). Now if $\mathscr{L}_{i}$ is a commutative subspace lattice on $\mathscr{H}_{i}$, we define $\otimes_{i=1}^{\infty_{a}} \mathscr{L}_{i} \subseteq \mathscr{B}(\mathscr{H})$ to be the smallest subspace lattice on $\mathscr{H}$ containing all the projections of the form $\otimes_{i=1}^{\infty} P_{i}$ with $P_{i} \in \mathscr{L}_{i}$ and $P_{i}=I$ for almost every $i$. We note that $\mathscr{H}$ has a basis consisting of elements of the form $\otimes_{i=1}^{\infty} h_{i}$ with $h_{i}=a_{i}$ for almost every $i[\mathbf{v N}]$. Thus, if $b_{i} \in \mathscr{H}_{i}^{\prime}$ with $\left\|b_{i}\right\|=1, i=1,2, \ldots$, then $\otimes_{i=1}^{\infty_{a}} \mathscr{H}_{i}$ and $\bigotimes_{i=1}^{\infty_{h}} \mathscr{H}_{i}^{\prime}$ are unitarily equivalent via a unitary operator constructed in the obvious way from unitaries $U_{i}$ : $\mathscr{H}_{i} \rightarrow \mathscr{H}_{i}^{\prime}$ with $U_{i}\left(a_{i}\right)=b_{i}$. Moreover, $\otimes_{i=1}^{\infty_{a}} \mathscr{L}_{i} \cong \otimes_{i=1}^{\infty_{b}} U_{i} \mathscr{L}_{i} U_{i}^{*}$.

Now consider lattices $\mathscr{L}\left(H_{i}, \leqslant_{i}, \mu_{i}\right)$ with $\mu_{i}\left(X_{i}\right)=1$. These lattices act on $L^{2}\left(X_{i}, \mu_{i}\right)$, and we use the constant function 1 as the distinguished unit vector in each $L^{2}\left(X_{i}, \mu_{i}\right)$ to form $\otimes_{i=1}^{\infty_{1}} L^{2}\left(X_{i}, \mu_{i}\right)$. Then $\otimes_{i=1}^{\infty_{1}} L^{2}\left(X_{i}, \mu_{i}\right)$ is canonically isomorphic to $L^{2}(X, \mu)$, where $X=\prod_{i=1}^{\infty} X_{i}$ and $\mu=\prod_{i=1}^{\infty} \mu_{i}$, and we will identify these two spaces in the sequel [G, Corollary 1.3]. The (isometric) isomorphism maps each element of the form $\otimes_{i=1}^{\infty} f_{i}$, where $f_{i} \equiv 1$ for almost every $i$, to the function $\prod_{i=1}^{\infty} f_{i}$ in $L^{2}(X, \mu)$.

THEOREM 3.8. Suppose $\mathscr{L}_{i}$ is a CSL acting on $\mathscr{H}_{i}$ and $a_{i}$ is a unit vector in $\mathscr{H}_{i}$, $i=1,2, \ldots$ Let $\mathscr{R}_{i}$ be a maximal abelian von Neumann algebra containing $\mathscr{L}_{i}^{\prime \prime}$, and let $M_{i}$ be the projection onto $\left[\mathscr{R}_{i} a_{i}\right]$, the closed linear span of $\left\{R_{i} a_{i}: R_{i} \in \mathscr{R}_{i}\right\}$. Then

(a) The tensor product of induced lattices $\otimes_{i=1}^{x_{a}}\left(\mathscr{L}_{i}\right)^{M_{i}}$ is unitarily equivalent to an infinite product lattice $\mathscr{L}(X, \leqslant, \mu)$. 
(b) If almost every $a_{i}$ is a cyclic vector for $\mathscr{R}_{i}$, then the full tensor product $\otimes_{i=1}^{\infty_{a}} \mathscr{L}_{i}$ is unitarily equivalent to an infinite product lattice.

Proof. $\mathscr{L}_{i}$ is unitarily equivalent to some $\mathscr{L}\left(Y_{i}, \leqslant_{i}, \nu_{i}\right)$ via the Arveson representation. An examination of the proof of the representation theorem (Theorem 1.3.1 of [A2]) shows that there is a unitary $U_{i}$ which maps $\left[\mathscr{R}_{i} c_{i}\right.$ ] onto some $L^{2}\left(Y_{i}, \nu_{i}\right)$, where $c_{i}$ is a cyclic vector for $\mathscr{R}_{i}$ (thus $\left.\left[\mathscr{R}_{i} c_{i}\right]=\mathscr{H}_{i}\right)$, and maps $c_{i}$ to the constant function 1 . By using $a_{i}$ instead of $c_{i}$, we get a unitary from $\left[\mathscr{R}_{i} a_{i}\right]=M_{i} \mathscr{H}_{i}$ onto some $L^{2}\left(X_{i}, \mu_{i}\right)$ which maps $a_{i}$ to the constant function 1. It then follows from the remainder of the proof that this unitary gives an equivalence between $\left(\mathscr{L}_{i}\right)^{M_{i}}$ and a lattice $\mathscr{L}\left(X_{i}, \leqslant{ }_{i}, \mu_{i}\right)$, and the above remarks imply that $\otimes_{i=1}^{\infty_{a}}\left(\mathscr{L}_{i}\right)^{M_{i}} \cong$ $\otimes_{i=1}^{\infty} \mathscr{L}\left(X_{i}, \leqslant{ }_{i}, \mu_{i}\right)$. For part (b), if $a_{i}$ is cyclic for $\mathscr{R}_{i}$ for all $i \geqslant$ some $N$, then apply the usual Arveson representation, using any cyclic vector for $i<N$ and $a_{i}$ for $i \geqslant N$. In this case we get a unitary equivalent between the full tensor product $\otimes_{i=1}^{\infty_{a}} \mathscr{L}_{i}$ and some $\otimes_{i=1}^{\infty_{1}} \mathscr{L}\left(X_{i}, \leqslant i, \mu_{i}\right)$.

Now form the infinite product lattice $\mathscr{L}(X, \leqslant, \mu)$ from the partially ordered measure spaces $\left(X_{i}, \leqslant{ }_{i}, \mu_{i}\right)$. To show that $\mathscr{L}(X, \leqslant, \mu)=\otimes_{i=1}^{\infty_{1}} \mathscr{L}\left(X_{i}, \leqslant{ }_{i}, \mu_{i}\right)$ (i.e., canonically isomorphic), we use a slight variation of the argument given in [GHL, Proposition 2.1]. First, for each $P_{i} \in \mathscr{L}\left(X_{i}, \leqslant_{i}, \mu_{i}\right)$, let $E_{i}$ be a corresponding increasing Borel subset of $X_{i}$. Then $E_{1} \times E_{2} \times \cdots \times E_{n} \times X_{n+1} \times X_{n+2} \times \cdots$ is an increasing Borel subset of $X$ which corresponds to the projection $P_{1} \otimes$ $P_{2} \otimes \cdots \otimes P_{n} \otimes I \otimes I \otimes \cdots$. It follows that $\otimes_{i=1}^{\infty_{1}} \mathscr{L}\left(X_{i}, \leqslant{ }_{i}, \mu_{i}\right) \subseteq \mathscr{L}(X, \leqslant, \mu)$ since $\otimes_{i=1}^{\infty_{1}} \mathscr{L}\left(X_{i}, \leqslant{ }_{i}, \mu_{i}\right)$ is generated by such projections.

Now for each $i$, let $\left\{E_{i k}: 1 \leqslant k<\infty\right\}$ be a sequence of increasing Borel subsets of $X_{i}$ such that $x_{i} \leqslant y_{i} \Leftrightarrow \chi_{E_{i k}}\left(x_{i}\right) \leqslant \chi_{E_{i k}}\left(y_{i}\right)$ for all $k$. Such sequences exist by [A2, Proposition 1.1.2]. The sets of the form $F_{i k}=X_{1} \times \cdots \times X_{i-1} \times E_{i k} \times X_{i+1} \times$ $X_{i+2} \times \cdots$ are increasing Borel subsets of $X$, and $x \leqslant y \Leftrightarrow \chi_{F_{i k}}(x) \leqslant \chi_{F_{i k}}(y)$ for all $i, k$. Let $S_{i k}$ be the projection in $\mathscr{L}(X, \leqslant, \mu)$ corresponding to $F_{i k}$. By [A2, Theorem 1.2.2], $\mathscr{L}(X, \leqslant, \mu)$ is generated by $\left\{S_{i k}\right\}$. But since $S_{i k}=I \otimes \cdots \otimes I \otimes$ $P_{i k} \otimes I \otimes I \otimes \cdots$, where $P_{i k}$ corresponds to $E_{i k}$, it follows that $\mathscr{L}(X, \leqslant, \mu) \subseteq$ $\otimes_{i=1}^{\infty_{1}} \mathscr{L}\left(X_{i}, \leqslant{ }_{i}, \mu_{i}\right)$.

THEOREM 3.9. (a) Suppose that $\mathscr{L}_{i}$ is a commutative subspace lattice on $\mathscr{H}_{i}$ and $a_{i}$ is a unit vector in $\mathscr{H}_{i}, i=1,2, \ldots$ If there is a sequence $\left\{P_{i} \in \mathscr{L}_{i}: 0<\left\|P_{i} a_{i}\right\|<1\right.$, $1 \leqslant i<\infty\}$ such that $\sum_{i=1}^{\infty}\left|\omega_{i}-\left\|P_{i} a_{i}\right\|^{2}\right|$ does not converge for every sequence $\left(\omega_{i}\right)$ of 0 's and 1's, then $\otimes_{i=1}^{\infty_{u}} \mathscr{L}_{i}$ is noncompact.

(b) Suppose that $\mathscr{L}(X, \leqslant, \mu)$ is an infinite product lattice. If there is a sequence $\left\{E_{i}: E_{i}\right.$ is an increasing Borel subset of $X_{i}$ and $\left.0<\mu_{i}\left(E_{i}\right)<1,1 \leqslant i<\infty\right\}$ such that $\sum_{i=1}^{\infty}\left|\omega_{i}-\mu_{i}\left(E_{i}\right)\right|$ does not converge for every sequence $\left(\omega_{i}\right)$ of 0 's and 1's, then $\mathscr{L}(X, \leqslant, \mu)$ is noncompact.

Note. In particular, if there is a sequence $\left\{P_{i} \in \mathscr{L}_{i}\right\}$ such that $\left\{\left\|P_{i} a_{i}\right\|\right\}$ is bounded away from 0 and 1 , then $\otimes_{i=1}^{\infty_{a}} \mathscr{L}_{i}$ is noncompact (and a similar condition applies in part (b)). 
Proof. (b) Let $F_{i}=\left\{\left(x_{i}\right)_{i=1}^{\infty}: x_{i} \in E_{i}\right\}$. It follows from the above example (Cases 2 and 3$)$ that the sublattice generated by $\left\{F_{i}: 1 \leqslant i<\infty\right\}$ is noncompact. By Proposition $3.3, \mathscr{L}(X, \leqslant, \mu)$ is also noncompact.

(a) Let $\mathscr{R}_{i}$ be a maximal abelian von Neumann algebra containing $\mathscr{L}_{i}^{\prime \prime}$, and let $M_{i}$ be the projection onto $\left[\mathscr{R}_{i} a_{i}\right]$. By Theorem $3.8, \bigotimes_{i=1}^{\infty_{a}}\left(\mathscr{L}_{i}\right)^{M_{i}}$ is unitarily equivalent to an infinite product lattice $\mathscr{L}(X, \leqslant, \mu)$. If $E_{i}$ is an increasing Borel subset of $X_{i}$ corresponding to $P_{i} M_{i}$, then

$$
\left\|P_{i} a_{i}\right\|^{2}=\left\|P_{i} M_{i} a_{i}\right\|^{2}=\int \chi_{E_{i}}\left(x_{i}\right) \cdot 1 d \mu_{i}\left(x_{i}\right)=\mu_{i}\left(E_{i}\right)
$$

(b) then implies that $\otimes_{i=1}^{\infty_{u}}\left(\mathscr{L}_{i}\right)^{M_{i}}$ is noncompact. But note that $\bigotimes_{i=1}^{\infty_{a}}\left(\mathscr{L}_{i}\right)^{M_{i}}$ is an induced lattice of $\otimes_{i=1}^{\infty_{a}} \mathscr{L}_{i}$, so $\otimes_{i=1}^{\infty_{a}} \mathscr{L}_{i}$ is noncompact by Proposition 3.3.

COROLlaRY 3.10. Every infinite tensor product of nonatomic CSLs is noncompact.

In fact, one could say that "most" infinite tensor products of CSLs are noncompact, since the $a_{i}$ 's would have to be chosen carefully in order to not satisfy the hypothesis of Theorem 3.9, especially if the $\mathscr{L}_{i}$ 's contain "lots" of projections. If the hypothesis of Theorem 3.9 is not satisfied, then the situation can be quite complicated. However, if almost every $a_{i}$ is cyclic for $\mathscr{R}_{i}$, then the nontrivial projections of $\mathscr{L}_{i}$ must "cluster" to 0 and/or $I$ as $i \rightarrow \infty$, and in the special case in which each $\mathscr{L}_{i}$ is a purely atomic nest, one can then show that $\otimes_{i=1}^{\infty_{a}} \mathscr{L}_{i}$ is purely atomic and thus compact (similar to Case 1 of the above example). On the other hand, of course, if any single $\mathscr{L}_{i}$ is noncompact, then so is $\otimes_{i=1}^{x_{a}} \mathscr{L}_{i}$ by Proposition 3.3.

It should be pointed out that infinite tensor products and nonatomic Boolean algebras (and generalizations using Proposition 3.3) are the only two types of noncompact CSLs known. In fact, they are also the only known examples of CSLs which are not completely distributive. Thus, to help settle the question of whether or not compact implies completely distributive, it would be desirable to have more examples.

4. Weak limits of projections. An examination of the proof of Theorem 2.2 shows that the full power of the hypotheses were never used. We did not need the invariance of the sequence $\left\{P_{n}\right\}$ (i.e., that $T P_{n}=P_{n} T P_{n}$ for all $T \in \mathscr{A}$ ). We only needed the property that $T P_{n}-P_{n} T P_{n} \rightarrow 0$ weakly for all $T \in \mathscr{A}$. Thus, it seems doubtful that the converse is true. However, in the commutative case, we can show that these properties are not that far apart after all. (Note also that in the commutative case it is enough to prove the converse for $\mathscr{L}$ nonatomic (see the proof of Corollary 2.3).) More precisely, consider the following properties, where $\mathscr{A}$ is a norm-closed subalgebra of $\mathscr{B}(\mathscr{H})$ and $Q \in \mathscr{B}(\mathscr{H})$ :

(a) There is a sequence $\left\{P_{n}\right\}$ of projections such that $T P_{n}-P_{n} T P_{n}=0$ for all $T \in \mathscr{A}$, and $P_{n} \rightarrow Q$ weakly.

(b) There is a sequence $\left\{Q_{n}\right\}$ of projections such that $\left\|T Q_{n}-Q_{n} T Q_{n}\right\| \rightarrow 0$ and $T Q_{n}-Q_{n} T Q_{n} \in \mathscr{K}$ for all $T \in \mathscr{A}$, and $Q_{n} \rightarrow Q$ weakly.

(c) There is a sequence $\left\{R_{n}\right\}$ of projections such that $\left\|T R_{n}-R_{n} T R_{n}\right\| \rightarrow 0$ for all $T \in \mathscr{A}$, and $R_{n} \rightarrow Q$ weakly. 
(d) There is a sequence $\left\{S_{n}\right\}$ of projections such that $T S_{n}-S_{n} T S_{n} \rightarrow 0$ weakly for all $T \in \mathscr{A}$, and $S_{n} \rightarrow Q$ weakly.

Note that $0 \leqslant Q \leqslant I$. If $\mathscr{A}$ is separable and unital, it was shown in [FPV] that (c) and (d) are equivalent. We will show in Theorem 4.1 below that, by simplifying the proof in [FPV], (b) is also equivalent. Finally, if $\mathscr{L}$ is a commutative nonatomic subspace lattice and $\mathscr{A}=$ alg $\mathscr{L}$, then (a) and (b) are equivalent (see Theorem 4.2).

The results of this section are essentially independent of the previous sections, but are related to them as indicated above and in Corollary 4.5 below. After obtaining the results noted above, we will give other conditions related to the presence of a weak limit $Q$ which satisfies property (c) (equivalently, (b) or (d)). These weak limits are completely characterized in the case when $\mathscr{A}$ is a $C^{*}$-algebra.

In this section, $\pi$ denotes the quotient map of $\mathscr{B}(\mathscr{H})$ onto the Calkin algebra $\mathscr{B}(\mathscr{H}) / \mathscr{K}$. Also, suppose $\mathscr{T}$ is a linear subspace of a unital $C^{*}$-algebra $\mathscr{B}$ (with $\mathscr{T}$ containing the identity), $\mathscr{M}_{n}=\{$ complex $n \times n$ matrices $\}$, and $\mathscr{H}^{(n)}=\mathscr{H} \oplus$ $\mathscr{H} \oplus \cdots \oplus \mathscr{H}(n$ times). If $\psi: \mathscr{T} \rightarrow \mathscr{B}(\mathscr{H})$ is a bounded linear map, then we define $\psi_{n}: \mathscr{T} \otimes \mathscr{M}_{n} \rightarrow \mathscr{B}\left(\mathscr{H}^{(n)}\right)$ by $\psi_{n}\left(\left(T_{i j}\right)_{i, j=1}^{n}\right)=\left(\psi\left(T_{i j}\right)\right)_{i, j=1}^{n} \cdot \psi$ is completely contractive if each $\psi_{n}$ is contractive, $\psi$ is completely isometric if each $\psi_{n}$ is isometric, and $\psi$ is completely bounded if there is some number $M$ such that $\left\|\psi_{n}\right\| \leqslant M$ for all $n$. We note that if $\psi$ is a unital $*$-homomorphism of a $C^{*}$-algebra, then $\psi$ is completely contractive. It follows that any restriction of $\psi$ to a linear subspace containing the identity is also completely contractive. In particular, $\pi$ is completely contractive on every subspace of $\mathscr{B}(\mathscr{H})$ which contains the identity $I$. For complete details, see $\$ 1.1$ and 1.2 of $[\mathbf{A 4}]$.

THEOREM 4.1. (except for (b), this is Theorem 2.1 of [FPV]. Let $\mathscr{A}$ be a norm-closed subalgebra of $\mathscr{B}(\mathscr{H})$ with $I \in \mathscr{A}$, and suppose $Q \in \mathscr{B}(\mathscr{H}), 0 \leqslant Q \leqslant I$. Then

$$
\text { property }(\mathrm{b}) \Rightarrow \text { property }(\mathrm{c}) \Rightarrow \text { property }(\mathrm{d})
$$

$\Rightarrow$ (e) There are two linear multiplicative mappings $\varphi: \mathscr{A} \rightarrow \mathscr{B}(\overline{Q \mathscr{H}})$ and $\theta: \pi(\mathscr{A}) \rightarrow \mathscr{B}(\overline{Q(I-Q) \mathscr{H}})$ uniquely defined by the relations

$$
\sqrt{Q} \varphi(T)=T \sqrt{Q} \mid \overline{Q \mathscr{H}} \text { and } \sqrt{I-Q} \varphi(T)=\theta(\pi(T)) \sqrt{I-Q} \mid \overline{Q \mathscr{H}},
$$

with $\theta$ completely contractive.

$\Rightarrow$ (f) There is a representation $\rho$ of $\pi\left(C^{*}(\mathscr{A})\right)$ on some separable Hilbert space $\mathscr{H}^{\prime}$, and a subspace $\mathscr{M} \subseteq \mathscr{H} \oplus \mathscr{H}^{\prime}$ invariant for $($ id $\oplus(\rho \circ \pi))(\mathscr{A})$, such that

$$
\left.P_{\mathscr{H}} P_{\mathscr{M}}\right|_{\mathscr{H}}=Q
$$

If $\mathscr{A}$ is norm-separable, then $(\mathrm{f}) \Rightarrow(\mathrm{b})$.

Proof. (b) $\Rightarrow$ (c) $\Rightarrow$ (d) is clear. (d) $\Rightarrow$ (e) $\Rightarrow$ (f) was aproved in [FPV] (separability of $\mathscr{A}$ was assumed, but not needed for these implications). (f) $\Rightarrow$ (c) was also proved in [FPV]. We will give a simpler proof which yields the stronger result (f) $\Rightarrow(\mathrm{b})$.

Let $\psi$ be the representation id $\oplus(\rho \circ \pi)$ of $C^{*}(\mathscr{A})+\mathscr{K}$ on the separable Hilbert space $\mathscr{H}_{1}=\mathscr{H} \oplus \mathscr{H}^{\prime}$. By Voiculescu's Theorem [V, Theorem 1.3], there is a sequence of unitary operators $U_{k}: \mathscr{H}_{1} \rightarrow \mathscr{H}$ such that

$$
U_{k} \psi(T) U_{k}^{*}-T \in \mathscr{K} \quad \text { and } \quad \lim _{k \rightarrow \infty}\left\|U_{k} \psi(T) U_{k}^{*}-T\right\|=0
$$


for all $T \in C^{*}(\mathscr{A})+\mathscr{K}$. Now let $q_{k} \in \mathscr{K}$ be any sequence of finite rank projections such that $q_{k} \rightarrow I$ strongly. By replacing $\left\{U_{k}\right\}$ by a subsequence, we can assume that

$$
\begin{gathered}
\left\|U_{k} \psi\left(q_{k} Q q_{k}\right) U_{k}^{*}-q_{k} Q q_{k}\right\|<1 / k \text { and } \\
\left\|U_{k} \psi\left(q_{k}\right) U_{k}^{*}-q_{k}\right\|<1 / k .
\end{gathered}
$$

Then

$$
\begin{aligned}
&\left\|q_{k}\left(U_{k} P_{\mathscr{M}} U_{k}^{*}-Q\right) q_{k}\right\| \\
& \leqslant\left\|U_{k} \psi\left(q_{k}\right) P_{\mathscr{M}} \psi\left(q_{k}\right) U_{k}^{*}-q_{k} Q q_{k}\right\| \\
&+\left\|q_{k} U_{k} P_{\mathscr{M}} U_{k}^{*} q_{k}-U_{k} \psi\left(q_{k}\right) P_{\mathscr{M}} \psi\left(q_{k}\right) U_{k}^{*}\right\| \\
& \leqslant\left\|U_{k} \psi\left(q_{k}\right) P_{\mathscr{M}} \psi\left(q_{k}\right) U_{k}^{*}-q_{k} Q q_{k}\right\|+\left\|\left(q_{k} U_{k}-U_{k} \psi\left(q_{k}\right)\right) P_{\mathscr{M}} U_{k}^{*} q_{k}\right\| \\
&+\left\|U_{k} \psi\left(q_{k}\right) P_{\mathscr{M}}\left(U_{k}^{*} q_{k}-\psi\left(q_{k}\right) U_{k}^{*}\right)\right\| \\
& \leqslant\left\|U_{k} \psi\left(q_{k}\right) P_{\mathscr{M}} \psi\left(q_{k}\right) U_{k}^{*}-q_{k} Q q_{k}\right\|+\left\|q_{k} U_{k}-U_{k} \psi\left(q_{k}\right)\right\| \\
&+\left\|U_{k}^{*} q_{k}-\psi\left(q_{k}\right) U_{k}^{*}\right\| \\
&=\left\|U_{k} \psi\left(q_{k}\right) P_{\mathscr{M}} \psi\left(q_{k}\right) U_{k}^{*}-q_{k} Q q_{k}\right\|+2\left\|U_{k} \psi\left(q_{k}\right) U_{k}^{*}-q_{k}\right\| \\
&<\left\|U_{k} \psi\left(q_{k}\right) P_{\mathscr{M}} \psi\left(q_{k}\right) U_{k}^{*}-q_{k} Q q_{k}\right\|+2 / k \quad(\text { by }(2)) \\
& \leqslant\left\|U_{k} \psi\left(q_{k}\right) P_{\mathscr{M}} \psi\left(q_{k}\right) U_{k}^{*}-U_{k} \psi\left(q_{k}\right)(Q \oplus 0) \psi\left(q_{k}\right) U_{k}^{*}\right\| \\
&+\left\|U_{k} \psi\left(q_{k}\right)(Q \oplus 0) \psi\left(q_{k}\right) U_{k}^{*}-q_{k} Q q_{k}\right\|+2 / k .
\end{aligned}
$$

But $\psi\left(q_{k}\right)=q_{k} \oplus 0$ since $q_{k} \in \mathscr{K}$, so

$$
\begin{aligned}
\psi\left(q_{k}\right) P_{\mathscr{M}} \psi\left(q_{k}\right) & \left(h \oplus h^{\prime}\right)=\left(q_{k} \oplus 0\right) P_{\mathscr{M}}\left(q_{k}(h) \oplus 0\right) \\
& =\left(q_{k} \oplus 0\right) P_{\mathscr{H}} P_{\mathscr{M}}\left(q_{k}(h) \oplus 0\right)+\left(q_{k} \oplus 0\right) P_{\mathscr{H}} P_{\mathscr{M}}\left(q_{k}(h) \oplus 0\right) \\
& =\left(q_{k} \oplus 0\right)(Q \oplus 0)\left(q_{k}(h) \oplus 0\right)+(0 \oplus 0) \quad\left(\text { since }\left.P_{\mathscr{H}} P_{\mathscr{M}}\right|_{\mathscr{H}}=Q\right) \\
& =q_{k} Q q_{k}(h) \oplus 0=\left(q_{k} \oplus 0\right)(Q \oplus 0)\left(q_{k} \oplus 0\right)\left(h \oplus h^{\prime}\right) \\
& =\psi\left(q_{k}\right)(Q \oplus 0) \psi\left(q_{k}\right)\left(h \oplus h^{\prime}\right) \\
& \Rightarrow \psi\left(q_{k}\right) P_{\mathscr{M}} \psi\left(q_{k}\right)-\psi\left(q_{k}\right)(Q \oplus 0) \psi\left(q_{k}\right)=0 \\
& \Rightarrow\left\|U_{k} \psi\left(q_{k}\right) P_{\mathscr{M}} \psi\left(q_{k}\right) U_{k}^{*}-U_{k} \psi\left(q_{k}\right)(Q \oplus 0) \psi\left(q_{k}\right) U_{k}^{*}\right\|=0 .
\end{aligned}
$$

Also,

$$
\begin{aligned}
\| U_{k} \psi\left(q_{k}\right) & (Q \oplus 0) \psi\left(q_{k}\right) U_{k}^{*}-q_{k} Q q_{k} \| \\
& =\left\|U_{k}\left(q_{k} Q q_{k} \oplus 0\right) U_{k}^{*}-q_{k} Q q_{k}\right\| \\
& =\left\|U_{k} \psi\left(q_{k} Q q_{k}\right) U_{k}^{*}-q_{k} Q q_{k}\right\|<1 / k \quad(\text { by }(2)) .
\end{aligned}
$$


Therefore, by (3), (4), and (5), it follows that

$$
\left\|q_{k}\left(U_{k} P_{\mathscr{M}} U_{k}^{*}-Q\right) q_{k}\right\|<0+1 / k+2 / k=3 / k .
$$

Now let $P_{k}=U_{k} P_{\mathscr{M}} U_{k}^{*}$. We first claim that $P_{k} \rightarrow Q$ weakly as $k \rightarrow \infty$. To see this, note that

$$
\begin{aligned}
\left\langle U_{k} P_{\mathscr{M}} U_{k}^{*} h,\right. & k\rangle-\langle Q h, k\rangle \\
= & \left\langle q_{k}\left(U_{k} P_{\mathscr{M}} U_{k}^{*}-Q\right) h, k\right\rangle+\left\langle\left(I-q_{k}\right)\left(U_{k} P_{\mathscr{M}} U_{k}^{*}-Q\right) h, k\right\rangle \\
= & \left\langle q_{k}\left(U_{k} P_{\mathscr{M}} U_{k}^{*}-Q\right) q_{k} h, k\right\rangle+\left\langle q_{k}\left(U_{k} P_{\mathscr{M}} U_{k}^{*}-Q\right)\left(I-q_{k}\right) h, k\right\rangle \\
& +\left\langle\left(I-q_{k}\right)\left(U_{k} P_{\mathscr{M}} U_{k}^{*}-Q\right) q_{k} h, k\right\rangle \\
& +\left\langle\left(I-q_{k}\right)\left(U_{k} P_{\mathscr{M}} U_{k}^{*}-Q\right)\left(I-q_{k}\right) h, k\right\rangle .
\end{aligned}
$$

The first term converges to 0 by (6). The second term converges to 0 since

$$
\begin{aligned}
\left|\left\langle q_{k}\left(U_{k} P_{\mathscr{M}} U_{k}^{*}-Q\right)\left(I-q_{k}\right) h, k\right\rangle\right| \leqslant\left\|\left(I-q_{k}\right) h\right\|\left\|\left(U_{k} P_{\mathscr{M}} U_{k}^{*}-Q\right) q_{k} k\right\| \\
\leqslant\left\|\left(I-q_{k}\right) h\right\|\left(\left\|P_{\mathscr{M}}\right\|+\|Q\|\right)\|k\| \leqslant 2\left\|\left(I-q_{k}\right) h\right\|\|k\|
\end{aligned}
$$

and $q_{k} \rightarrow 1$ strongly. The third and fourth terms converge to 0 by similar computations.

Next, for $T \in \mathscr{A}$ we have

$$
\begin{aligned}
\left\|\left(I-P_{k}\right) T P_{k}\right\|=\left\|U_{k}\left(I-P_{\mathscr{M}}\right) U_{k}^{*} T U_{k} P_{\mathscr{M}} U_{k}^{*}\right\| \\
\quad=\left\|U_{k}\left(I-P_{\mathscr{M}}\right)\left(U_{k}^{*} T U_{k}-\psi(T)+\psi(T)\right) P_{\mathscr{M}} U_{k}^{*}\right\| \\
\quad \leqslant\left\|U_{k}\left(I-P_{\mathscr{M}}\right)\left(U_{k}^{*} T U_{k}-\psi(T)\right) P_{\mathscr{M}} U_{k}^{*}\right\|+\left\|U_{k}\left(I-P_{\mathscr{M}}\right) \psi(T) P_{\mathscr{M}} U_{k}^{*}\right\| \\
\quad \leqslant\left\|U_{k}^{*} T U_{k}-\psi(T)\right\|+\left\|U_{k}\left(I-P_{\mathscr{M}}\right) \psi(T) P_{\mathscr{M}} U_{k}^{*}\right\| \\
\quad=\left\|T-U_{k} \psi(T) U_{k}^{*}\right\| \quad(\text { since } \mathscr{M} \text { is invariant for }(i d \oplus \rho \circ \pi)(\mathscr{A})=\psi(\mathscr{A})) \\
\quad \rightarrow 0 \quad(\text { by }(1)) .
\end{aligned}
$$

Finally, if $T \in \mathscr{A}$, then

$$
\begin{aligned}
\left(I-P_{k}\right) T P_{k} & =U_{k}\left(I-P_{\mathscr{M}}\right) U_{k}^{*} T U_{k} P_{\mathscr{M}} U_{k}^{*} \\
& =U_{k}\left(I-P_{\mathscr{M}}\right)\left(U_{k}^{*} T U_{k}-\psi(T)+\psi(T)\right) P_{\mathscr{M}} U_{k}^{*} \\
& =U_{k}\left(I-P_{\mathscr{M}}\right)\left(U_{k}^{*} T U_{k}-\psi(T)\right) P_{\mathscr{M}} U_{k}^{*}+U_{k}\left(I-P_{\mathscr{M}}\right) \psi(T) P_{\mathscr{M}} U_{k}^{*} .
\end{aligned}
$$

But again the second term equals 0 by the invariance of $\mathscr{M}$, and the first term is compact since $T-U_{k} \psi(T) U_{k}^{*} \in \mathscr{K}$ by (1). Therefore, $\left(I-P_{k}\right) T P_{k} \in \mathscr{K}$ for all $k$.

THEOREM 4.2. If $\mathscr{L}$ is a commutative nonatomic subspace lattice and $\mathscr{A}=\operatorname{alg} \mathscr{L}$, then properties (a) and (b) are equivalent.

For the proof, we will need two additional results.

TheOREM 4.3 [Da1, TheOREM 1.1]. If $\mathscr{L}$ is a CSL and $\mathscr{A}=\operatorname{alg} \mathscr{L}$, then $\pi(\mathscr{L})=$ $\{p \in \mathscr{B}(\mathscr{H}) / \mathscr{K}: p$ is a projection and $(1-p) \pi(T) p=0$ for every $T \in \mathscr{A}\}$. 
Theorem 4.4 [An, Proposition 1.4.2 and Corollary 1.4.3]. Let $\mathscr{R}$ be a type I von Neumann algebra on $\mathscr{H}$ and let $\mathscr{D} \subseteq \mathscr{B}(\mathscr{H})$ be a set of operators. Let $\delta$ be a positive function on $\mathscr{D}$ such that for each $R \in \mathscr{R}$ and $D \in \mathscr{D}$, we have $D R-R D \in \mathscr{K}$ and $\left\|D_{n} R-R D_{n}\right\| \rightarrow 0$ whenever $\delta\left(D_{n}\right) \rightarrow 0$. Then there are operators $P_{D} \in \mathscr{R}^{\prime}$ such that $P_{D}-D \in \mathscr{K}$ and $\left\|P_{D_{n}}-D_{n}\right\| \rightarrow 0$ whenever $\delta\left(D_{n}\right) \rightarrow 0$. If $D$ is a projection, then $P_{D}$ can be chosen to be a projection.

Proof of Theorem 4.2. The implication $(a) \Rightarrow(b)$ is clear, so assume (b) is true. If $\left\{Q_{n}\right\}$ is finite, then the result is trivial, so we may assume $\left\{Q_{n}\right\}$ is infinite. Now $\mathscr{L}^{\prime \prime}$ is an abelian von Neumann algebra, so $\mathscr{L}^{\prime}$ is a type I von Neumann algebra. Let $\mathscr{R}=\mathscr{L}^{\prime}, \mathscr{D}=\left\{Q_{n}\right\}$, and $\delta\left(Q_{n}\right)=1 / n$. Then the hypotheses of Theorem 4.4 are satisfied since $\mathscr{L}^{\prime} \subseteq \mathscr{A}$ and $\mathscr{L}^{\prime}$ is selfadjoint. Let $P_{n}=P_{Q_{n}}$ be the projections in $\mathscr{L}^{\prime \prime}$ provided by Theorem 4.4. Then $P_{n} \rightarrow Q$ weakly since $Q_{n} \rightarrow Q$ weakly and $\left\|P_{n}-Q_{n}\right\| \rightarrow 0$.

Now note that $\kappa\left(Q_{n}\right) \in \kappa(\mathscr{L})$ by Theorem 4.3. It follows that there is a projection $S_{n} \in \mathscr{L}$ such that $Q_{n}-S_{n} \in \mathscr{K}$, so $P_{n}-S_{n} \in \mathscr{L}^{\prime \prime} \cap \mathscr{K}$. But $P_{n}$ and $S_{n}$ are projections and $\mathscr{L}^{\prime \prime}$ is nonatomic, so we must have $P_{n}=S_{n}$. Therefore, $P_{n} \in \mathscr{L}$.

We immediately obtain a stronger version of Theorem 2.2 .

COROllary 4.5. Let $\mathscr{A}=\operatorname{alg} \mathscr{L}$. If $\mathscr{L}$ is noncompact, then there is a nonprojection $Q$ which satisfies properties (e) and (f).

We now turn to the study of the relationship between an algebra $\mathscr{A}$ and a weak limit $Q$ which satisfies properties (b), (c), and (d). In addition to Corollary 4.5 and the remarks at the beginning of this section, this will give an indication of the obstructions involved in investigating the converse of Theorem 2.2. As in [FPV], we say that $Q$ is a weak limit of almost invariant projections for $\mathscr{A}$, or WLP. (We will use the notation WLP to mean that $Q$ is nontrivial (not 0 or $I$ ), but it may be a projection.) In the remainder of this section, $\mathscr{A}$ denotes a norm-closed, unital, norm-separable subalgebra of $\mathscr{B}(\mathscr{H})$. We also say that $Q$ is a weak limit of almost reducing projections for $\mathscr{A}$ if $Q$ is a WLP for both $\mathscr{A}$ and $\mathscr{A}^{*}$. It is easy to see that this is equivalent to $Q$ being a WLP for $C^{*}(\mathscr{A})$, and that in this case we must have that $Q \in \mathscr{A}^{\prime}$ (by property (d)).

REMARK. If $Q$ is a WLP, then $T P_{n}-P_{n} T P_{n} \rightarrow 0$ and $P_{n} \rightarrow Q$ weakly, so the proof of Theorem 2.2 shows that $\overline{Q \mathscr{H}}$ and $\operatorname{ker}(I-Q)$ are invariant subspaces for $\mathscr{A}$, $\operatorname{ker}(I-Q) \subseteq \overline{Q \mathscr{H}}$, and $(I-Q) K Q=0$ (i.e., $K(\overline{Q \mathscr{H}}) \subseteq \operatorname{ker}(I-Q))$ for $K \in \mathscr{A} \cap$ $\mathscr{K}$. The following results are almost immediate.

Proposition 4.6. Suppose $\mathscr{A}$ has a $W L P Q$. Then

(i) $\overline{(I-Q) Q \mathscr{H}} \subseteq \cap\left\{\operatorname{ker} K: K \in \mathscr{A} \cap \mathscr{A}^{*} \cap \mathscr{K}\right\}$.

(ii) If $\operatorname{ker}(I-Q)=\{0\}$, then $\overline{Q \mathscr{H}} \subseteq \bigcap\{\operatorname{ker} K: K \in \mathscr{A} \cap \mathscr{K}\}$.

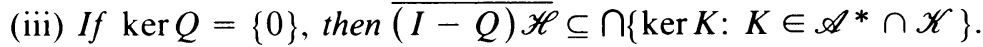

(iv) If $\operatorname{ker} Q=\operatorname{ker}(I-Q)=\{0\}$, then $\mathscr{A} \cap \mathscr{K}=\{0\}$.

(v) If $Q \in \mathscr{A}^{\prime}$, then $\overline{(I-Q) Q \mathscr{H}} \subseteq \bigcap\left\{\operatorname{ker} K: K \in \mathscr{A} \cap \mathscr{K}\right.$ or $\left.K \in \mathscr{A}^{*} \cap \mathscr{K}\right\}$. 
Proof. (ii) and (iv) are immediate from the above remark. (i) and (iii) follow by noting that $Q$ is a WLP for $\mathscr{A}$ iff $I-Q$ is a WLP for $\mathscr{A}^{*}$. Finally, if $Q \in \mathscr{A}^{\prime}$, then $\operatorname{ker}(I-Q), \overline{(I-Q) Q \mathscr{H}}$, and $\operatorname{ker} Q$ are reducing subspaces for $\mathscr{A}$, and (v) follows from the above remark.

COROllary 4.7. If $\mathscr{A}$ has a WLP, then either (i) $\mathscr{A}$ has a nontrivial closed invariant subspace or (ii) $\mathscr{A} \cap \mathscr{K}=\{0\}$.

COROLlaRy 4.8. If $\mathscr{A}$ has a weak limit of almost reducing projections, then either (i) $\mathscr{A}$ is reducible or (ii) $C^{*}(\mathscr{A}) \cap \mathscr{K}=\{0\}$.

Corollary 4.9. If $\mathscr{K} \subseteq \mathscr{A}$, then $\mathscr{A}$ has no WLP. Thus, if $\mathscr{A}$ is transitive ( $\mathscr{A}$ has no nontrivial closed invariant subspace) and $\mathscr{A}$ contains a nonzero compact operator, then $\mathscr{A}$ has no WLP.

Proof. By [PS, Theorem 7], a transitive algebra which contains a nonzero compact operator must contain all the compact operators.

We can now completely characterize the weak limits of almost reducing projections of $\mathscr{A}$ (i.e., the WLPs of $C^{*}(\mathscr{A})$ ).

THEOREM 4.10. $Q$ is a WLP for $C^{*}(\mathscr{A}) \Leftrightarrow Q=I_{\mathscr{L}} \oplus Q_{\mathscr{M}} \oplus 0_{\mathscr{N}}$, where $\mathscr{L}, \mathscr{M}$, and $\mathscr{N}$ are reducing subspaces for $\mathscr{A}$ with $\mathscr{L} \oplus \mathscr{M} \oplus \mathscr{N}=\mathscr{H}, \mathscr{M}=\bigcap\{\operatorname{ker} K: K \in$ $\left.C^{*}(\mathscr{A}) \cap \mathscr{K}\right\}$, and $Q_{\mathscr{M}} \in\left(\left.C^{*}(\mathscr{A})\right|_{\mathscr{M}}\right)^{\prime}, 0_{\mathscr{M}} \leqslant Q_{\mathscr{M}} \leqslant I_{\mathscr{M}}$.

Proof. $\Rightarrow$ Suppose $Q$ is a WLP for $C^{*}(\mathscr{A})$. Recall that $Q \in\left(C^{*}(\mathscr{A})\right)^{\prime}$. Let $\mathscr{L}^{\prime}=\operatorname{ker}(I-Q), \mathscr{M}^{\prime}=\overline{(I-Q) Q \mathscr{H}}$, and $\mathscr{N}^{\prime}=\operatorname{ker} Q$. Then $\mathscr{M}^{\prime} \subseteq \bigcap\{\operatorname{ker} K: K \in$ $\left.C^{*}(\mathscr{A}) \cap \mathscr{K}\right\}$ by Proposition 4.6(v). Let $\mathscr{M}=\bigcap\left\{\operatorname{ker} K: K \in C^{*}(\mathscr{A}) \cap \mathscr{K}\right\} . \mathscr{M}$ is invariant for $C^{*}(\mathscr{A})$, so it reduces $\mathscr{A}$. It also follows that $Q_{\mathscr{M}} \in\left(\left.C^{*}(\mathscr{A})\right|_{\mathscr{M}}\right)^{\prime}$, where $Q_{\mathscr{M}}=\left.Q\right|_{\mathscr{M}}$. Now let $\mathscr{L}=\mathscr{L}^{\prime} \cap \mathscr{M}^{\perp}$ and $\mathscr{N}=\mathscr{N}^{\prime} \cap \mathscr{M}^{\perp}$. Then $\mathscr{L}$ and $\mathscr{N}$ are reducing subspaces for $\mathscr{A}, \mathscr{L} \oplus \mathscr{M} \oplus \mathscr{N}=\mathscr{H}$ since $\mathscr{L}^{\prime} \oplus \mathscr{M}^{\prime} \oplus \mathcal{N}^{\prime}=\mathscr{H}$, and $Q=I_{\mathscr{L}} \oplus Q_{\mathscr{M}} \oplus 0_{\mathscr{N}}$

$\Leftarrow$ Define $\bar{\theta}: \pi\left(C^{*}(\mathscr{A})\right) \rightarrow \mathscr{B}(\mathscr{M})$ by $\bar{\theta}(\pi(T))=\left.T\right|_{\mathscr{M}}$. Then $\bar{\theta}$ is well defined by the definition of $\mathscr{M}$ and is completely contractive since it is a $*$-homomorphism. Therefore, $\theta: \quad \pi\left(C^{*}(\mathscr{A})\right) \rightarrow \mathscr{B}\left(\overline{(I-Q) Q \mathscr{H})}\right.$ by $\theta(\pi(T))=\left.T\right|_{(I-Q) Q \mathscr{H}}$ is completely contractive since $\overline{(I-Q) Q \mathscr{H}} \subseteq \mathscr{M}$. Now let $\varphi: C^{*}(\mathscr{A}) \rightarrow \mathscr{B}(\overline{Q \mathscr{H}})$ by $\varphi(T)=T \mid \overline{Q \mathscr{H}}$ and note that the identities in Theorem 4.1(e) are satisfies since $\sqrt{Q}$ and $\sqrt{I-Q}$ commute with all $T$ in $C^{*}(\mathscr{A})$.

REMARK. The assumption that $\mathscr{A}$ is unital can be easily dealt with in this case. If $\mathscr{A}$ is nonunital, let $\mathscr{A}_{1}=\left\{A+\lambda I: A \in C^{*}(\mathscr{A})\right\}$ be the unital $C^{*}$-algebra associated with $\mathscr{A}$. Then it is easy to see that $Q$ is a WLP for $C^{*}(\mathscr{A})$ iff it is a WLP for $\mathscr{A}_{1}$.

Corollary 4.11. If $C^{*}(\mathscr{A}) \cap \mathscr{K}=\{0\}$, then every positive contraction $Q$ in $\mathscr{A}^{\prime}$ is a $W L P$ for $C^{*}(\mathscr{A})$.

COROLlaRY 4.12. If the identity representation of $C^{*}(\mathscr{A})$ is not a direct sum of irreducible representations, then $C^{*}(\mathscr{A})$ has a nonprojection $W L P$. 
Proof. If $C^{*}(\mathscr{A})$ has no nonprojection WLP, then Theorem 4.10 implies that $\cap\left\{\operatorname{ker} K: K \in C^{*}(\mathscr{A}) \cap \mathscr{K}\right\}=\{0\}$. It follows that the identity representation of $C^{*}(\mathscr{A}) \cap \mathscr{K}$ is nondegenerate and is thus a direct sum of irreducible representations. These representations then extend uniquely to $C^{*}(\mathscr{A})[\mathbf{A 1}, \S 1.3-1.4]$.

Minor variations of the proof of Theorem 4.10 allow us to characterize the WLPs of $\mathscr{A}$ which lie in $\mathscr{A}^{\prime}$ :

THeORem 4.13. $Q$ is a WLP for $\mathscr{A}$ and $Q \in \mathscr{A}^{\prime} \Leftrightarrow Q=I_{\mathscr{L}} \oplus Q_{\mathscr{M}} \oplus 0_{\mathscr{N}}$, where $\mathscr{L}$, $\mathscr{M}$, and $\mathscr{N}$ are reducing subspaces for $\mathscr{A}$ with $\mathscr{L} \oplus \mathscr{M} \oplus \mathscr{N}=\mathscr{H}, Q_{\mathscr{M}} \in\left(\left.\mathscr{A}\right|_{\mathscr{M}}\right)^{\prime}$, and $0_{\mathscr{M}} \leqslant Q_{\mathscr{M}} \leqslant I_{\mathscr{M}}$, and the map $\delta:\left.\pi(T) \rightarrow T\right|_{\mathscr{M}}$ is well-defined and completely contractive on $\pi(\mathscr{A})$.

Proof. $\Rightarrow$ It follows from the fact that $Q \in \mathscr{A}^{\prime}$ that the maps $\varphi$ and $\theta$ of Theorem 4.1(e) are defined by $\varphi(T)=T \mid \overline{Q_{\mathscr{H}}}$ and $\theta(\pi(T))=T \mid \overline{(I-Q) Q \mathscr{H}}$. Now let $\mathscr{M}=\overline{(I-Q) Q \mathscr{H}}, \mathscr{L}=\operatorname{ker}(I-Q), \mathscr{N}=\operatorname{ker} Q$, and $\delta=\theta$. The result follows since $\theta$ is completely contractive.

$\Leftarrow$ It is clear that $Q \in \mathscr{A}^{\prime}$. Now note that the map $\theta: \pi(T) \rightarrow T \mid \overline{(I-Q) Q \mathscr{H}}$ defined on $\pi(\mathscr{A})$ is completely contractive since $\overline{(I-Q) Q \mathscr{H}} \subseteq \mathscr{M}$. By defining $\varphi(T)=\left.T\right|_{Q \mathscr{E}}$ for $T \in \mathscr{A}$, the conditions of Theorem 4.1(e) hold since $Q \in \mathscr{A}^{\prime}$. Thus $Q$ is a WLP for $\mathscr{A}$.

Corollary 4.14. Suppose that $Q \in \mathscr{A}^{\prime}, 0 \leqslant Q \leqslant I$. Then $Q$ is a WLP for $\mathscr{A} \Leftrightarrow$ the map $\theta: \pi(T) \rightarrow T \mid \overline{(I-Q) Q \mathscr{H}}$ is well-defined and completely contractive on $\pi(\mathscr{A})$.

REMARK. The corollary shows that if $Q \in \mathscr{A}^{\prime}$ is a WLP for $\mathscr{A}$, then $I-Q$ is also a WLP for $\mathscr{A}$, so $Q$ is also a WLP for $\mathscr{A}^{*}$. However, the sequence of projections corresponding to $Q$ as a WLP for $\mathscr{A}^{*}$ may be different than the sequence of projections corresponding to $Q$ as a WLP for $\mathscr{A}$. In particular, $Q$ may not be a weak limit of reducing projections, as shown in Example 3 below.

COROllary 4.15. If $\overline{\mathscr{A}+\mathscr{A}^{*}}=C^{*}(\mathscr{A})$ and $Q \in \mathscr{A}^{\prime}$ is a WLP for $\mathscr{A}$, then $Q$ is also a weak limit of reducing projections for $\mathscr{A}$.

Proof. By Corollary 4.14, the map $\theta: \pi(T) \rightarrow T \mid \overline{(I-Q) Q \mathscr{H}}$ is completely contractive and unital. The obvious extension to $\overline{\pi(\mathscr{A})+\pi(\mathscr{A})^{*}}=\pi\left(C^{*}(\mathscr{A})\right)$ is then completely contractive by $[\mathbf{A 4}, 1.2 .8-1.2 .10]$, and another application of Corollary 4.14 gives the result.

Corollary 4.16. Suppose $\mathscr{A} \cap \mathscr{K}=\{0\}$. If $\pi: \mathscr{A} \rightarrow \mathscr{B}(\mathscr{H}) / \mathscr{K}$ is completely isometric, then every positive contraction $Q$ in $\mathscr{A}^{\prime}$ is a WLP for $\mathscr{A}$. If $\pi$ is not completely isometric, then $\mathscr{A}$ has no WLP $Q$ in $\mathscr{A}^{\prime}$ with $\operatorname{ker} Q=\operatorname{ker}(I-Q)=\{0\}$.

ProOF. If $\pi$ is completely isometric, then $\theta=\pi^{-1}$ is completely contractive. The results then follow from Corollary 4.14.

EXAMPLE 1 . If $Q$ is a WLP for $C^{*}(\mathscr{A})$, one might expect that $Q \mid \overline{(I-Q) Q \mathscr{H}}$ is a WLP for $C^{*}(\mathscr{A} \mid \overline{(I-Q) Q \mathscr{H}})$. However, this need not be true since the projections in the sequence for $Q$ do not necessarily commute with the projection onto $\overline{(I-Q) Q \mathscr{H}}$. For a specific example, suppose $T \in \mathscr{B}(\mathscr{H})$ such that $C^{*}(T) \supseteq \mathscr{K}$ (for instance, $T$ 
could be the unilaterial shift). Then $C^{*}(T)$ has no WLPs by Corollary 4.8. Now let $T_{0}=T \oplus T \oplus T \oplus \cdots$, acting on $\mathscr{H}_{0}=\mathscr{H} \oplus \mathscr{H} \oplus \cdots$ Then $C^{*}\left(T_{0}\right)$ contains no compact operators, so every positive contraction in $C^{*}\left(T_{0}\right)^{\prime}$ is a WLP for $C^{*}\left(T_{0}\right)$ by Corollary 4.11. Thus, $Q=\lambda I \oplus 0 \oplus 0 \oplus \cdots, 0<\lambda<1$, is a WLP for $C^{*}\left(T_{0}\right)$. But $\overline{(I-Q) Q \mathscr{H}_{0}}=\mathscr{H} \oplus 0 \oplus 0 \oplus \cdots$, so $\left.Q\right|_{(I-Q) Q \mathscr{H}_{0}}=\lambda I_{\mathscr{H}}$ and $\left.T_{0}\right|_{(I-Q) Q \mathscr{H}_{0}}=T$. Therefore, $Q \mid \overline{(I-Q) Q \mathscr{H}_{0}}$ is not a WLP for $C^{*}\left(T_{0} \mid \overline{(I-Q) Q \mathscr{H}_{0}}\right)$.

EXample 2. Again, suppose $T \in \mathscr{B}(\mathscr{H})$ such that $C^{*}(T) \supseteq \mathscr{K}$, and this time let $T_{0}=T \oplus T$. Then $\cap\left\{\operatorname{ker} K: K \in C^{*}\left(T_{0}\right) \cap \mathscr{K}\right\}=\{0\}$, but $C^{*}\left(T_{0}\right)$ does not contain \{compact operators on $\mathscr{H} \oplus \mathscr{H}\}$. Thus, $C^{*}\left(T_{0}\right)$ has no nonprojection WLP by Theorem 4.10, and the only WLPs are the projections which commute with $C^{*}\left(T_{0}\right)$.

In a similar manner, we can obtain a partial converse to Corollary 4.12. If the identity representation of a $C^{*}$-algebra $\mathscr{A}$ is a direct sum of irreducible representations $\sigma_{i}$, and each $\sigma_{i}(\mathscr{A})$ contains a compact operator (and thus all compacts), then $\mathscr{A}$ has no nonprojection WLP.

EXAMPLE 3. Let $S$ be the unilateral shift, i.e., $S e_{n}=e_{n+1}$ for an orthonormal basis $\left\{e_{1}, e_{2}, \ldots\right\}$ of $\mathscr{H} . S$ is irreducible and $S S^{*}-S^{*} S \in \mathscr{K}$, so $S$ is essentially normal and $C^{*}(S) \supseteq \mathscr{K}$. Let $\mathscr{A}(S)$ be the norm-closed algebra generated by $S$ and $I$. Then the map $\pi: \mathscr{A}(S) \rightarrow \mathscr{B}(\mathscr{H}) / \mathscr{K}$ is completely isometric by [A5, Corollary 2 , p. 292], so $C^{*}(S)$ has no WLPs but $\mathscr{A}(S)$ has many WLPs (every $\lambda I, 0<\lambda<1$, is a WLP for $\mathscr{A}(S)$ by Corollary 4.16$)$. This yields an easy proof that $\mathscr{A}(S)+\mathscr{A}(S)^{*}$ is not dense in $C^{*}(S)$, just by applying Corollary 4.15 .

EXAMPLE 4. Now suppose that $W$ is a unilateral weighted shift, $W e_{n}=\omega_{n} e_{n+1}$, with $\omega_{n} \neq 0, \lim \sup \left|\omega_{n}\right|<\sup \left|\omega_{n}\right|$, and $\left|\omega_{n+1}\right|-\left|\omega_{n}\right| \rightarrow 0$ as $n \rightarrow \infty$. Then $W$ is again essentially normal, irreducible, and $C^{*}(W) \supseteq \mathscr{K}$, but this time $\pi: \mathscr{A}(W) \rightarrow$ $\mathscr{B}(\mathscr{H}) / \mathscr{K}$ is not completely isometric [A5, Theorem 2.2.1]. Thus, $\mathscr{A}(W)$ has no WLPs in $\mathscr{A}(W)^{\prime}$ by Theorem 4.13. In this case, one must look for WLPs not in $\mathscr{A}(W)^{\prime}$.

We turn now to the case in which $Q$ is a WLP for $\mathscr{A}$ but $Q \notin \mathscr{A}^{\prime}$. If $Q$ and $I-Q$ have closed range, then we can obtain a stronger necessary condition than that given by Theorem 4.1(e).

Theorem 4.17. Suppose $Q$ is a WLP for $\mathscr{A}$ such that $Q$ and $I-Q$ have closed range. Let $\mathscr{M}=(I-Q) Q \mathscr{H}$. Then the map $\delta: \pi(\mathscr{A}) \rightarrow \mathscr{B}(\mathscr{M})$ by $\delta(\pi(T))=$ $P_{\mathscr{M}} T P_{\mathscr{M}}$ is completely bounded, where $P_{\mathscr{M}}$ is the projection onto $\mathscr{M}$.

Proof. Theorem 4.1(e) implies that there are maps $\theta$ and $\varphi$ such that

$$
\sqrt{Q} \theta(\pi(T)) \sqrt{I-Q} \sqrt{Q} h=\sqrt{Q} \sqrt{I-Q} \varphi(T) \sqrt{Q} h=\sqrt{I-Q} T Q h,
$$

and it follows that

$$
\begin{aligned}
\langle\theta(\pi(T)) \sqrt{I-Q} \sqrt{Q} h, \sqrt{I-Q} \sqrt{Q} k\rangle & =\langle\sqrt{I-Q} T Q h, \sqrt{I-Q} k\rangle \\
& =\langle(I-Q) T Q h, k\rangle .
\end{aligned}
$$

If we let $P=P_{\mathscr{M}}$ and $Q^{\prime}=\left.Q\right|_{\mathscr{M}}$, then $Q^{\prime}$ and $P-Q^{\prime}$ are invertible in $\mathscr{B}(\mathscr{M})$. For $s, t \in \mathscr{M}$, the above identity applied to $h=\sqrt{P-Q^{\prime}} \sqrt{Q^{\prime}} s$ and $k=$ ${\sqrt{P-Q^{\prime}}}^{-1} \sqrt{Q^{\prime}}{ }^{-1} t$ yields $\langle\theta(\pi(T)) s, t\rangle=\left\langle\sqrt{P-Q^{\prime}} \sqrt{Q^{\prime}}-1\right.$ T $\left.\sqrt{Q^{\prime}}{\sqrt{P-Q^{\prime}}}^{-1} s, t\right\rangle$. 
Now if $x_{j}, y_{i} \in \mathscr{H}$ and $\left(T_{i j}\right) \in \mathscr{A} \otimes \mathscr{M}_{n}$, we let $u_{j}=\sqrt{P-Q^{\prime}}{\sqrt{Q^{\prime}}}^{-1} P x_{j}$ and $v_{i}$ $=\sqrt{Q^{\prime}} \sqrt{P-Q^{\prime}}-1 P y_{i}$ and obtain

$$
\begin{aligned}
& \left|\sum_{i, j=1}^{n}\left\langle P T_{i j} P x_{j}, y_{i}\right\rangle\right|=\left|\sum\left\langle T_{i j} P x_{j}, P y_{i}\right\rangle\right| \\
& =\left|\sum\left\langle T_{i j} \sqrt{Q^{\prime}} \sqrt{P-Q^{\prime}}{ }^{-1} u_{j}, \sqrt{P-Q^{\prime}}{\sqrt{Q^{\prime}}}^{-1} v_{i}\right\rangle\right|=\left|\sum\left\langle\theta\left(\pi\left(T_{i j}\right)\right) u_{j}, v_{i}\right\rangle\right| \\
& \leqslant\left\|\left(\pi\left(T_{i j}\right)\right)\right\|\left\|\oplus u_{j}\right\|\left\|\oplus v_{i}\right\| \quad \text { (since } \theta \text { is completely contractive) } \\
& \leqslant\left\|\left(\pi\left(T_{i j}\right)\right)\right\|\left\|\sqrt{P-Q^{\prime}} \sqrt{Q^{\prime}}-1\right\|\left\|\sqrt{Q^{\prime}} \sqrt{P-Q^{\prime}}-1\right\|\left\|\oplus x_{j}\right\|\left\|\oplus y_{i}\right\| \\
& \Rightarrow \delta \text { is completely bounded by } M=\left\|\sqrt{P-Q^{\prime}}{\sqrt{Q^{\prime}}}^{-1}\right\|\left\|\sqrt{Q^{\prime}} \sqrt{P-Q^{\prime}}-1\right\| \text {. }
\end{aligned}
$$

Question. Suppose conversely that there is a semi-invariant projection $P_{\mathscr{M}}$ for $\mathscr{A}$ such that the map $\delta: \pi(T) \rightarrow P_{\mathscr{M}} T P_{\mathscr{M}}$ is completely bounded by some $M(M$ is necessarily $\geqslant 1)$. Is there some positive contraction $Q^{\prime} \in \mathscr{B}(\mathscr{M})$ such that $Q^{\prime}$ and $P_{\mathscr{M}}-Q^{\prime}$ are invertible in $\mathscr{B}(\mathscr{M})$ and such that the map $\theta: \pi(\mathscr{A}) \rightarrow \mathscr{B}(\mathscr{M})$ by $\theta(\pi(T))={\sqrt{P_{\mathscr{M}}-Q^{\prime}}}_{Q^{\prime}}^{-1} T \sqrt{Q^{\prime}}{\sqrt{P_{\mathscr{M}}-Q^{\prime}}}^{-1}$ is completely contractive? If so, then it follows that $\mathscr{A}$ has a WLP $Q$. To see this, let $P_{\mathscr{M}}=P_{1}-P_{0}$, where $P_{1}$ and $P_{0}$ are invariant projections for $\mathscr{A}$ and $P_{1}>P_{0}$, and define $Q$ by $Q=I_{P_{0} \mathscr{H}} \oplus Q^{\prime} \oplus 0_{P_{1}^{\perp} \mathscr{H}}$. Then by reversing the first part of the argument in the proof of Theorem 4.17 , it follows that $\langle\theta(\pi(T)) \sqrt{I-Q} \sqrt{Q} h, \sqrt{I-Q} \sqrt{Q} k\rangle=\langle T Q h,(I-Q) k\rangle$ for $h, k \in$ $\mathscr{H}$. Now define $\varphi: \mathscr{A} \rightarrow \mathscr{B}(\overline{Q \mathscr{H}})$ by $\langle\varphi(T) \sqrt{Q} h, \sqrt{Q} k\rangle=\langle T Q h, k\rangle$. It is easy to check that $\varphi$ is linear and multiplicative and that $\varphi$ and $\theta$ satisfy the identities of Theorem 4.1(e).

To simplify matters, suppose that $\delta: \pi(T) \rightarrow T$ is completely bounded on $\pi(\mathscr{A})$ by $M \geqslant 1$. Then we want to find a positive contraction $Q$ such that $\theta: \pi(T)$ $\rightarrow \sqrt{I-Q} \sqrt{Q}-1 T \sqrt{Q} \sqrt{I-Q^{-1}}$ is completely contractive. This is equivalent to finding a positive invertible operator $A$ such that $\pi(T) \rightarrow A^{-1} T A$ is completely contractive. If $\delta$ is completely isometric, then this is equivalent to finding an $A$ such that $T \rightarrow A^{-1} T A$ is completely contractive. In this case $\mathscr{A}$ has many WLPs in $\mathscr{A}^{\prime}$ by Corollary 4.16 , but if $A \notin \mathscr{A}^{\prime}$ this would yield a WLP $Q \notin \mathscr{A}^{\prime}$. For example, if $S$ is the unilateral shift and $\mathscr{A}=\mathscr{A}(S)$, is there some positive invertible $A \notin \mathscr{A}^{\prime}$ such that $T \rightarrow A^{-1} T A$ is completely contractive on $\mathscr{A}$ ?

\section{REFERENCES}

[A1] W. B. Arveson, An invitation to $C^{*}$-algebras, Graduate Texts in Math., no. 39, Springer-Verlag, Berlin and New York, 1976.

[A2] __. Operator algebras and invariant subspaces, Ann. of Math. (2) 100 (1974), 433-532.

[A3] _ Perturbation theory for groups and lattices, J. Funct. Anal. 53 (1983), 22-73.

[A4] _. Subalgebras of $C^{*}$-algebras, Acta Math. 123 (1969), 141-224.

[A5] _. Subalgebras of $C^{*}$-algebras. II, Acta Math. 128 (1972), 271-308.

[An] N. T. Andersen, Compact perturbations of reflexive algebras, J. Funct. Anal. 38 (1980), 366-400.

[Da1] K. R. Davidson, Commutative subspace lattices, Indiana Univ. Math. J. 27 (1978), 479-490.

[Da2] . Similarity and compact perturbations of nest algebras, J. Reine Angew. Math. 348 (1984), $72-87$. 
[Di] J. Dixmier, Les algèbres d'opérateurs dans l'espace Hilbertien, 2nd ed., Gauthier-Villars, 1969.

[FAM] T. Fall, W. Arveson, and P. Muhly, Perturbations of nest algebras, J. Operator Theory 1 (1979), 137-150.

[FPV] C. Foias, C. Pasnicu, and D. Voiculescu, Weak limits of almost invariant projections, J. Operator Theory 2 (1979), 79-93.

[F1] J. Froelich, Compact operators in the algebra of a partially ordered measure space, J. Operator Theory 10 (1983), 353-355.

[F2] _ Compact operators, invariant subspaces, and spectral synthesis, Doctoral Dissertation, Univ. of Iowa, 1984

[GHL] F. Gilfeather, A. Hopenwasser, and D. Larson, Reflexive algebras with finite width lattices: tensor products, cohomology, compact perturbations, J. Funct. Anal. 55 (1984), 176-199.

[G] A. Guichardet, Produits tensoriels infinis et représentations des relations d'anticommutation, Ann. Sci. École Norm. Sup. 83 (1966), 1-52.

[HLM] A. Hopenwasser, C. Laurie, and R. Moore, Reflexive algebras with completely distributive subspace lattices, J. Operator Theory 11 (1984), 91-108.

[Lam] M. S. Lambrou, Approximants, commutants and double commutants in normed algebras, J. London Math. Soc. (2) 25 (1982), 499-512.

[La] D. R. Larson, Nest algebras and similarity transformations, Ann. of Math. (2) 121 (1985), 409-427.

[L1] C. Laurie, On density of compact operators in reflexive algebras, Indiana Univ. Math. J. 30 (1981), $1-16$.

[L2] , Complete distributivity and ordered group lattices, Proc. Amer. Math. Soc. 95 (1985), 79-82.

[LL] C. Laurie and W. Longstaff, A note on rank-one operators in reflexive algebras, Proc. Amer. Math. Soc. 89 (1983), 293-297.

[PS] C. Pearcy and A. Shields, A survey of the Lomonosov technique in the theory of invariant subspaces, Topics in Operator Theory, Math. Surveys, no. 13, Amer. Math. Soc., Providence, R. I., 1974.

[R] W. Rudin, Functional analysis, McGraw-Hill, 1973.

[S] D. E. Sarason, On spectral sets having connected complement, Acta Sci. Math. (Szeged) 26 (1965), 289-299

[V] D. Voiculescu, A non-commutative Weyl-von Neumann theorem, Rev. Roumaine Math. Pures Appl. 212 (1976), 97-113.

[vN] J. von Neumann, On infinite direct products, Comp. Math. 6 (1938), 1-77.

Department of Mathematics, Iowa State University, Ames, Iowa 50011 